\title{
A comparison of parent-child interaction therapy and treatment as usual with families with a history of child abuse and neglect and intimate partner violence
}

\author{
Kimberly P. Foley \\ West Virginia University
}

Follow this and additional works at: https://researchrepository.wvu.edu/etd

\section{Recommended Citation}

Foley, Kimberly P., "A comparison of parent-child interaction therapy and treatment as usual with families with a history of child abuse and neglect and intimate partner violence" (2010). Graduate Theses, Dissertations, and Problem Reports. 3104.

https://researchrepository.wvu.edu/etd/3104

This Dissertation is protected by copyright and/or related rights. It has been brought to you by the The Research Repository @ WVU with permission from the rights-holder(s). You are free to use this Dissertation in any way that is permitted by the copyright and related rights legislation that applies to your use. For other uses you must obtain permission from the rights-holder(s) directly, unless additional rights are indicated by a Creative Commons license in the record and/ or on the work itself. This Dissertation has been accepted for inclusion in WVU Graduate Theses, Dissertations, and Problem Reports collection by an authorized administrator of The Research Repository @ WVU.

For more information, please contact researchrepository@mail.wvu.edu. 
A Comparison of Parent-Child Interaction Therapy and Treatment as Usual with Families with a History of Child Abuse and Neglect and Intimate Partner Violence

Kimberly P. Foley, MA, MS

Dissertation submitted to the Eberly College of Arts and Sciences

at West Virginia University in partial fulfillment of the requirements

for the degree of

Doctor of Philosophy

In

Psychology

Cheryl B. McNeil, PhD, Chair

Daniel Hursh, PhD

Elisa Krackow, PhD

Kevin Larkin, $\mathrm{PhD}$

Leslie E. Tower, $\mathrm{PhD}$

Department of Psychology

Morgantown, West Virginia

2010 


\begin{abstract}
A Comparison of Parent-Child Interaction Therapy and Treatment as Usual with Families with a History of Child Abuse and Neglect and Intimate Partner Violence
\end{abstract}

Kimberly P. Foley, MA, MS

Child abuse and neglect (CAN) and intimate partner violence (IPV) are serious public health issues that need to be addressed. In 2005, a reported 899,000 children throughout the United States, the District of Columbia, and Puerto Rico were the victims of either abuse or neglect resulting in the death of 1,460 children under 18-years-of-age (US Department of Health and Human Services, 2005). In the United States each year, adult women are subjected to over 5 million IPV incidences and which result in the death of approximately 1,300 women (APA, 2005). A meta-analysis of 31 studies conducted by Appel and Holden (1998) examining the cooccurrence of IPV and CAN revealed that these two forms of violence range from $20 \%-100 \%$ co-morbidity with an average of $41 \%$ co-morbidity. PCIT is an evidence-based treatment that has both theoretical and scientific support as a treatment to reduce future CAN recidivism and decrease the short- and long-term effects of CAN on children (Borrego et al., 1999; Chaffin et al., 2004; Timmer et al., 2005a; Timmer et al., 2005b, Timmer et al., 2006). Although PCIT has received theoretical support in the literature as a potential treatment for IPV (Borrego, Gutow, Reicher, \& Barker, 2008; Pearl, 2008), to date, no research has been conducted that has assessed the impact of PCIT upon IPV. Currently, no studies have been conducted to examine the effectiveness of PCIT in a community-based environment with co-morbid CAN and IPV populations. This study aimed to contribute to the fields of CAN, IPV, and PCIT by evaluating the effectiveness of PCIT with populations who experience co-morbid family violence. Analyses indicated that physical child abuse scores did have a greater decrease from pre- to post-treatment in the PCIT condition compared to the TAU condition, $F(2,41)=12.10, p=0.00$. Furthermore, this study provided some support for the extra benefits of PCIT over standard TAU treatment in terms of increasing parenting skill, decreasing child behavior problems, and decreasing parental stress. It provided preliminary support for providing PCIT in a community setting without additional equipment and rooms. Analyses also indicated that IPV did not have a greater decrease from pre- to post-treatment in the PCIT condition compared to the TAU conduction for male-to-female psychological violence, $F(2,39)=0.68, p=0.51$, female-to-male psychological violence, $F(2,39)=1.78, p=0.18$, male-to-female physical violence, $F(2,39)=0.49, p=0.61$, and female-to-male physical violence, $F(2,39)=0.93, p=0.40$. However, power and methodological problems made it difficult to interpret the findings. More studies need to be conducted exploring the impact of PCIT upon IPV to more accurately assess this program as a potential treatment for the reduction of co-morbid family violence. Limitations, implications, and future directions are also elucidated. 


\section{Acknowledgements}

Many individuals and organizations are owed a heartfelt thank you for their invaluable assistance and guidance throughout the duration of this project.

First, I would like to say thank you to Cheryl McNeil, $\mathrm{PhD}$ who allowed me to undertake such an extensive project and provided me with support during all stages and aspects of this project. Her supervision and guidance have made this journey more like an adventure.

Secondly, I would like to thank the other members of my thesis committee; Steven A. Branstetter, PhD, Dan Hursh, PhD, Elisa Krackow, PhD, Kevin Larkin, PhD, and Leslie Tower, $\mathrm{PhD}$. In your own individual way you have all contributed significantly to the conceptualization and implementation of this project. I hope you are as excited to read this document as I was to write it!

Not to be forgotten, Rachel Canning, Kayla Frederick, Brittany Glass, William Lemieux, Ciera Pauley, and Monica Waitkus deserve endless accolades for their long-term commitment to this project. They drove hundreds, if not thousands of miles, ate entirely too much of my cooking, scored lengthy questionnaires, endured hours of data entry, and worked with fabulous but challenging - families, all for the sake of a program that they believed would make a difference in the lives of participants. It truly could not have happened without all of you, thank you tremendously for your assistance.

I would like to express my sincere gratitude to the staff and families at The Family Junction, Inc. Their dedication to providing services to families in their community made this project possible. In particular, I would like to thank Keri B. Whitacre, MS, who was my coding partner in crime and comrade through the duration of this study. In addition, Melanie McDonald and Colleen Lowman also played a crucial role in helping with various aspects of this project.

Lastly, I would like to send loving thanks to my grandparents, William and N. Patricia Foley, and husband, S. Ramani Pillai, who have supported me throughout this long endeavor. I would not be the person I am today without their love and encouragement. To honor them, as they have honored me, I would like to dedicate this document to them. 
Table of Contents

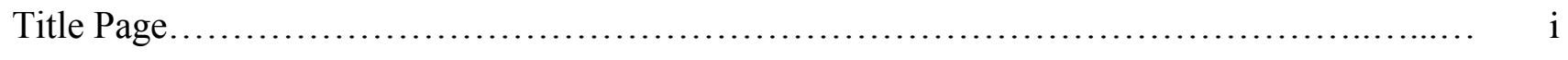

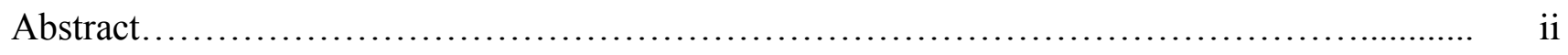

Acknowledgments................................................................

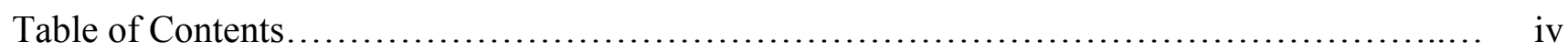

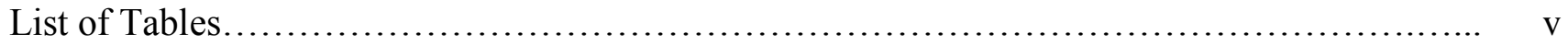

List of Appendixes............................................................ vi

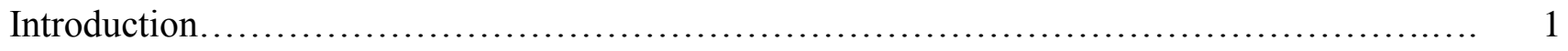

Child Abuse and Neglect..................................................... 1

Treatments for Child Abuse and Neglect....................................... 4

Theoretical Underpinnings of Parent-Child Interaction Therapy........................ 7

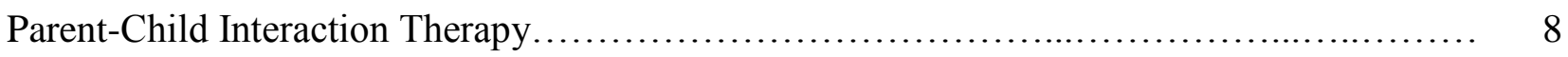

Unique Components and Strengths of Parent-Child Interaction Therapy.................. 12

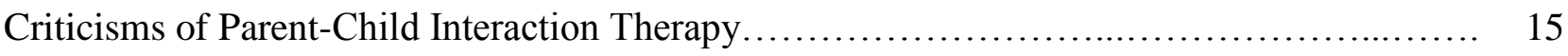

Parent-Child Interaction Therapy and Child Abuse and Neglect Populations................. 17

Parent-Child Interaction Therapy and Intimate Partner Violence........................ 22

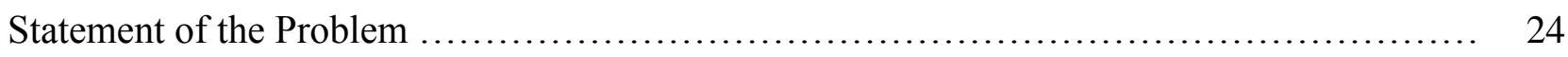

Hypotheses............................................................. 26

Methods..................................................................... 28

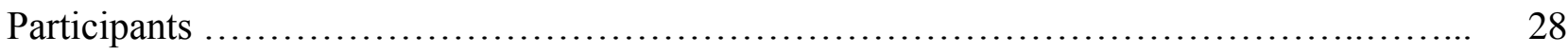

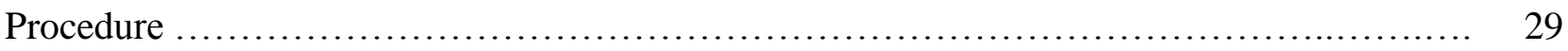

Parent-Child Interaction Therapy Condition...................................... 32 
Modifications for Individualized PCIT Format vs. Group PCIT Format.................... 35

Treatment as Usual Condition............................................... 36

Treatment Fidelity and Quality Assurance............................................ 37

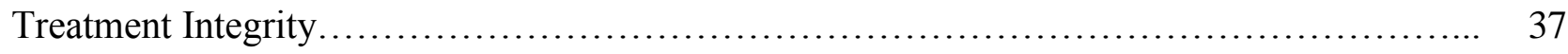

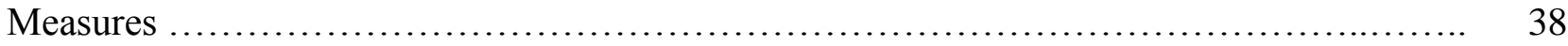

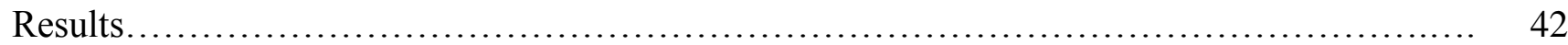

Preliminary Analyses........................................................ 42

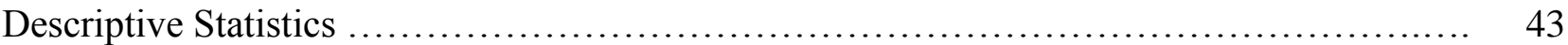

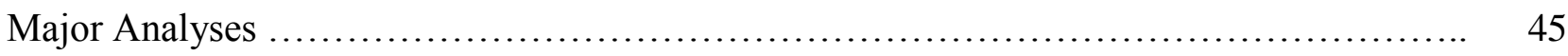

Exploratory Analyses............................................................ 57

Discussion................................................................... 59

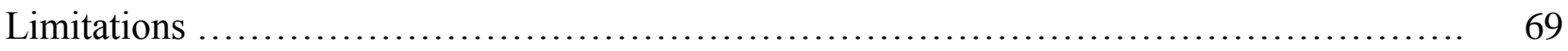

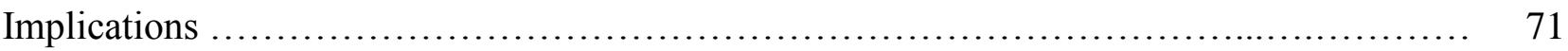

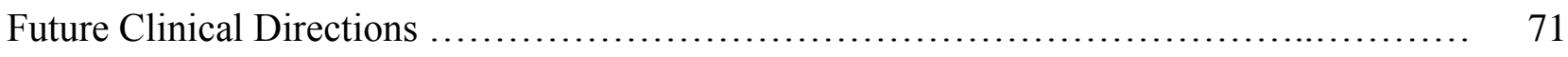

Future Research Directions ................................................... 73

References.................................................................. 75 
List of Tables

Table 1. Demographic data of participants.................................. 85

Table 2. Overall means of assessment measures.............................. 87

Table 3. ANOVA of CAP physical child abuse for PCIT and TAU.................. 88

Table 4. ANOVA of CTS2 female psychological violence for PCIT and TAU........... 89

Table 5. ANOVA of CTS2 male psychological violence for PCIT and TAU.......... 90

Table 6. ANOVA of CTS2 female physical violence for PCIT and TAU............. 91

Table 7. ANOVA of CTS2 male physical violence for PCIT and TAU.............. 92

Table 8. ANOVA of PSI-SF total stress for PCIT and TAU....................... 93

Table 9. ANOVA of CBCL internalizing behaviors for PCIT and TAU .............. 94

Table 10. ANOVA of CBCL externalizing behaviors for PCIT and TAU .............. 95

Table 11. ANOVA of CBCL total behaviors for PCIT and TAU................... 96

Table 12. Description of DPICS-III Child Compliance for PCIT and TAU............ 97

Table 13. ANOVA of ECBI Intensity for PCIT and TAU ............................ 98

Table 14. ANOVA of ECBI Problem for PCIT and TAU.......................... 99

Table 15. Paired samples t-tests of DPICS-III Positive Talk for PCIT .................. 100

Table 16. Paired samples t-tests of DPICS-III Negative Talk for PCIT................ 101

Table 17. Overall CTS2 scores........................................... 102 


\section{List of Appendices}

Appendix A: Demographic Form......................................... 103

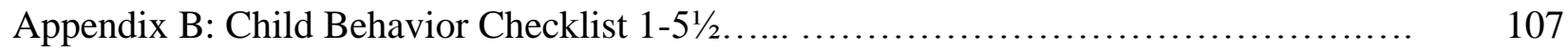

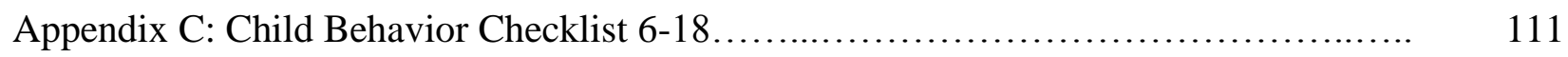

Appendix D: The Revised Conflict Tactics Scale............................... 115

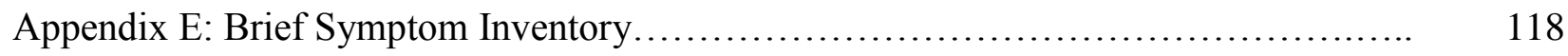

Appendix F: Child Abuse Potential Inventory-IV .............................. 122

Appendix G: Eyberg Child Behavior Inventory............................... 126

Appendix H: Dyadic Parent-Child Interaction Therapy Coding System-III............. 128

Appendix I: Parent Stress Index - Short Form.............................. 129

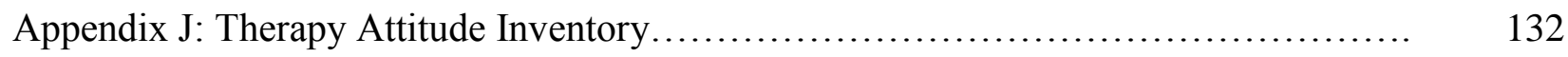


A Comparison of Parent-Child Interaction Therapy and Treatment as Usual with Families with a History of Child Abuse and Neglect and Intimate Partner Violence

\author{
Introduction
}

\title{
Child Abuse and Neglect
}

Child abuse and neglect (CAN) is a serious public health issue that needs to be addressed. A reported 899,000 children throughout the United States, the District of Columbia, and Puerto Rico were the victims of either abuse or neglect during 2005. Of these nearly 900,000 children, 564,572 experienced neglect, 149,234 experienced physical abuse, 83,607 experienced sexual abuse, and 63,829 experienced emotional or psychological abuse (US Department of Health and Human Services, 2005). Additionally, 1,460 children under 18-years-of-age died in 2005 due to abuse or neglect with $90 \%$ of these children under 7-years-of-age (US Department of Health and Human Services, 2005). Moreover, $54.5 \%$ of the victims of child maltreatment were also under 7-years-of-age (US Department of Health and Human Services, 2005). Furthermore, large-scale research studies have estimated the prevalence of CAN in the general population ranges from $11.4 \%$ (Springer, Sheridan, Kuo, \& Carnes, 2007) to 17.6\% (Boden, Horwood, \& Fergusson, 2007). These statistics demonstrate that CAN is rampant in the United States and that younger children are at an elevated risk to suffer the greatest from the effects of CAN (Lieberman \& Knorr, 2007).

Traditionally, CAN encompasses four major forms of child maltreatment: neglect, emotional abuse, physical abuse, and sexual abuse (US Department of Health and Human Services, 2005). The most thorough definition of CAN includes any act (or the failure to act) committed by an adult caretaker inflicted upon a minor child, which includes, but is not limited to, child emotional abuse (i.e., verbal bullying, failure to attend to emotional needs adequately), 
child neglect (i.e., possessing the ability or means to provide for a child but refusing to do so), child physical abuse (i.e., hitting, punching, slapping), and child sexual abuse (i.e., molestation, rape). While these forms of maltreatment may occur in isolation, it is more likely that a child actually experiences co-morbid forms of maltreatment (Herrenkohl \& Herrenkohl, 2007). For example, a child may experience simultaneous neglect and physical abuse.

Incidences of CAN are not typically singular, isolated occurrences. Rather, CAN are destructive patterns of behaviors to which children are exposed on numerous occasions for extended periods of time. Indeed, in a longitudinal study of over 1,000 18- and 21-year-olds, $17.6 \%$ of participants reported that their caregivers regularly or frequently used physical punishment (Boden et al., 2007). Furthermore, one of the most challenging components to treating families with a history of CAN is that these families tend to experience high recidivism rates, ranging from 19 to $49 \%$, subsequent to intervention or treatment (Chaffin, Silovsky, Funderbunk, Valle, Brestan, Alachua, et al., 2004; DePanfilis \& Zuravin, 2002). Hence, it is crucial that evidence-based treatment programs are developed and implemented in order to determine which programs are the most successful in providing the best therapeutic treatment for these families and reducing CAN recidivism.

A commonly held belief is that the majority of perpetrators of CAN towards children are strangers who are unknown to the child or family. This is an unsubstantiated claim, and, in truth, nearly $80 \%$ of the perpetrators of CAN are biological caregivers. Of this $80 \%$, approximately $91 \%$ of these caregivers are biological parents, $4 \%$ are step-parents, and $1 \%$ are adoptive caregivers (US Department of Health and Human Services, 2005). Additionally, female caregivers $(57.8 \%)$ were more likely to commit child maltreatment than male caregivers $(42.2 \%)$ (US Department of Health and Human Services, 2005). As the data have demonstrated, it is 
therefore important to specifically target maternal, biological caregivers when developing evidence-based treatments for CAN treatment programs.

The short- and long-term effects of CAN have been shown to have detrimental effects on children physically, emotionally, behaviorally, and psychologically (Cohen, Mannarino, Murray, \& Igelman, 2006; Herschell \& McNeil, 2005; Lieberman \& Knorr, 2007; Springer et al., 2007). Compared to children without a history of CAN, children with a history of CAN are at an increased risk for physical violence, sexual violence (Arias, 2004; Lang, Stein, Kennedy, \& Foy, 2004), and intimate partner violence (IPV) during adulthood (Arias, 2004). Children with a history of CAN are more likely to experience internalizing and externalizing behavior problems (Sternberg, et al., 2006), increased fear, inconsolable crying, temper tantrums, sleep disturbances, aggression, and withdrawal (Lieberman \& Knorr, 2007). During adulthood, these children are at an increased risk to suffer from psychopathology (Cohen et al., 2006; Lang et al., 2004), particularly anger, anxiety, and depression (Springer et al., 2007; Sternberg, Lamb, Guterman, \& Abbott, 2006). Overall, these children achieve less academically (Boden et al., 2007), are at an increased risk of suicide, are more likely to use alcohol and other substances (Makhija, 2007), and have elevated rates of high blood pressure, heart problems, and ulcers (Springer et al., 2007).

Early intervention may be an important factor in the reduction of the short- and long-term effects of CAN (Arias, 2004). The early identification of children who have experienced CAN or are at risk of experiencing CAN allows for treatments to be implemented at an early age. Early intervention increases the probability of reducing the effects of CAN and promises a better future for these children. More specifically, early intervention programs may: (a) decrease the duration 
of time a child experiences CAN, (b) target the caregivers most likely to implement CAN, (c) reduce CAN recidivism, and (d) decrease the effects of CAN upon children and families.

\section{Treatments for Child Abuse and Neglect}

According to the US Department of Health and Human Services (2007), two types of services are provided to families who are referred to child welfare services: preventative and post-investigation. Preventative services are offered to at-risk caregivers and children prior to documented CAN incidents. These services are both educational and therapeutic and include providing caregivers with information regarding developmentally appropriate behaviors for children and how to improve the quality of the caregiver-child relationship (US Department of Health and Human Services, 2007). Post-investigation services are offered to caregivers and children subsequent to substantiated CAN incidents to ensure the child's future safety. These services include family assessment regarding previous, present, and future CAN incidents, as well as providing educational and therapeutic services to reduce or eliminate potential future CAN (US Department of Health and Human Services, 2007).

Many children with a history of CAN do not receive any form of psychological treatment (Cohen et al., 2006). However, caregivers who have inflicted CAN may be court-ordered to receive post-investigative services in an attempt to reduce future $\mathrm{CAN}$ incidences. The majority of these caregivers receive services from community therapists who have not been trained to provide evidence-based practices (Cohen et al., 2006). In order to determine and provide the best services available for these families, organizations and researchers have focused their efforts towards reviewing the treatments that are currently available for these populations.

The Kauffman Best Practices Project (Chadwick, 2004) was developed to identify the best evidence-based practices available to assist children and families in healing from the effects 
of multiple forms of CAN. Stringent criteria were developed to ascertain which treatments would be considered the most efficacious. These criteria included having a sound theoretical basis, acceptance in clinical practices, evidence-based research in support of treatment effects, no known risk of harm to clients, a minimum of one randomized trial, and evidence of treatment efficacy. Furthermore, each treatment was required to have a treatment manual, to be able to be delivered with a reasonable degree of fidelity, and to be able to be delivered by trained, supervised professions. Based on these standards, three interventions were identified as best practices: Trauma Focused-Cognitive Behavioral Therapy (TF-CBT), Abuse Focused-Cognitive Behavioral Therapy (AF-CBT), and Parent-Child Interaction Therapy (PCIT). PCIT was selected for inclusion as a best practice for three reasons: research has demonstrated that PCIT (a) improves parenting skills, (b) decreases child behavior problems, and (c) improves the parent-child relationship.

Cohen and colleagues (2006) conducted another review to determine the best evidencebased practices available for children and families with a history of CAN. They too delineated several criteria to determine the most-sound interventions currently available. These criteria required that children (and not only caregivers) be actively involved in treatment, that child mental health be assessed, and that at least one randomized control trial had been conducted. Based on these criteria, 13 practices were identified as best practices: Trauma Focused-Cognitive Behavior therapy (TF-CBT), Psychodynamic/Psychoanalytic Treatment for Sexual Abuse, Abuse Focused-Cognitive Behavioral Therapy (AF-CBT), Child-Parent Psychotherapy (CPP), Behavioral Treatments for Children Exposed to Domestic Violence, Youth Relationships Project (YRP), Kids Club, Cognitive Behavioral Interventions for Trauma in Schools (CBITS), Resilient Peer Treatment, Multisystemic Therapy (MST), Multifamily Group Therapy (MFGT), Cognitive 
Behavioral Therapy for Childhood Traumatic Grief (CBT-CTG), and Trauma and Grief Focused Interventions for Adolescents. This inventory of evidence-based practices consists of treatments for CAN as well as other forms of violence to which children may be exposed. Due to the authors' criteria, PCIT was not included on this list of evidence-based practices because child mental health had not been previously assessed in studies with CAN populations. However, the research regarding PCIT and CAN was reviewed and the authors acknowledged that PCIT is an intervention program that reduces recidivism rates by targeting parenting behaviors.

PCIT was originally designed to be an early intervention program (Hembree-Kigin \& McNeil, 1995; Heschell \& McNeil, 2005) but has since evolved into an EBT which is able to provide post-investigation services to children and families with a history of CAN (Chaffin et al., 2004). There is ample theoretical support for PCIT as a treatment for CAN (Bell \& Eyberg, 2002; Herschell \& McNeil, 2005; McNeil, Filcheck, Greco, Ware, \& Bernard, 2001; Urquiza \& McNeil, 1996) and several studies have demonstrated success regarding the application of PCIT to CAN populations (Borrego, Urquiza, Rasmussen, \& Zebell, 1999; Chaffin et al., 2004; Dombrowski, Timmer, Blacker, \& Urquiza, 2005; Timmer, Uriquiza, \& Zebell, 2005a; Timmer, Urquiza, Zebell, \& McGrath, 2005b; Timmer, Urquiza, Herschell, McGrath, Zebell, Porter, et al., 2006). However, only Chaffin and colleagues (2004) have conducted a randomized control trial study examining PCIT with a CAN population and these results have yet to be replicated in community-based programs. As such, the development and implementation of community-based research studies is the next logical step to determine if PCIT will be successful treating CAN populations outside of a university setting. 


\section{Theoretical Underpinnings of Parent-Child Interaction Therapy}

PCIT is strongly grounded within several theoretical orientations: Baumrind's parenting model (1967), Bowlby and Ainsworth's attachment theory, traditional play therapy, and social learning theory (Eyberg, 1988; Niec, Hemme, Yopp, \& Brestan, 2005). According to Baumrind's model, parenting styles are directly related to child outcome. Baumrind (1967) determined three types of parenting styles (authoritarian, authoritative, and permissive) with each characterized by different amounts of parental warmth and control. Baumrind's research indicated that children with parents who met their child's emotional needs while also setting age-appropriate limits were more likely to develop into well-adjusted adolescents. PCIT incorporates this theory into its treatment program by promoting an authoritative parenting style which supports healthy child development (Bell \& Eyberg, 2002; Hembree-Kigin \& McNeil, 1995).

According to Bowlby and Ainsworth's model, parent-child attachment is also directly related to child outcome. Together, Bowlby and Ainsworth (1991) developed four types of parent-child relationships (secure-, avoidant-, ambivalent-, and disorganized-attachment) with each characterized by different parent-child interaction patterns. Bowlby and Ainsworth's research indicated that the early relationships children developed with their caregivers were indicative of the relationships they would develop with others in later life. Children with secure attachment styles were more likely to feel safe exploring their environment compared to those with avoidant-, ambivalent-, or disorganized-attachment who were less likely to attain healthy adolescent development. A main component of PCIT is to foster the development of a secure parent-child attachment which supports healthy child development (Eyberg, 1988).

Social learning theory postulates that individuals' behaviors are learned through the direct observations of people's behaviors and the contingencies those behaviors yield. Patterson 
(1982) expanded upon this framework and developed a coercion hypothesis which delineates how maladaptive behaviors are developed and maintained through differential reinforcement. CAN may result from a coercive parent-child relationship in which both the caregiver and child exhibit verbal behaviors that gradually escalate and may culminate into violent verbal or physical behavior (Brinkmeyer \& Eyberg, 2003). Maladaptive parent-child interactions are therefore a result of the reinforcement of these behaviors and may be modified by reinforcing more adaptive behaviors. PCIT addresses these maladaptive interaction patterns and instructs caregivers on how to positively reinforce adaptive behaviors, while decreasing maladaptive behaviors. Skills that are taught during the second phase of PCIT are derived from social learning theory and include components such as social reinforcement, positive and negative punishment, and the development of problem-solving skills (Niec et al., 2005).

\section{Parent-Child Interaction Therapy}

Parent-Child Interaction Therapy (PCIT) was developed in the 1970's by Shelia Eyberg, PhD. Eyberg's program was strongly influenced by the work of Constance Hanf, PhD, (1969) who developed a two-phase treatment model that addressed behavior problems in young children with multiple disabilities (Eyberg, 2004). During the first phase of Hanf's model, maternal caregivers were instructed on how to increase their child's positive behaviors while simultaneously decreasing their child's negative behaviors. In the second phase of Hanf's model, maternal caregivers were instructed on how to deliver clear and effective commands to their child coupled with positive reinforcement to increase adaptive child behaviors and negative punishment to decrease maladaptive child behaviors. Additionally, maternal caregivers were taught how to implement non-violent discipline techniques for child non-compliance and maladaptive behaviors. Hanf originally developed this model for mothers of "multi-handicapped 
children," however, PCIT has evolved from this initial framework and is presently utilized with such diverse populations as CAN (Borrego et al., 1999; Chaffin et al., 2004; Timmer et al., 2005a), fathers (Bagner \& Eyberg, 2003), siblings (Brestan, Eyberg, Boggs, \& Alginia, 1997), conduct disorder (Brestan \& Eyberg, 1998), oppositional defiant disorder (Brinkmeyer \& Eyberg, 2003; Calzada, Eyberg, Rich, \& Querido, 2004; Harwood \& Eyberg, 2004), Puerto Rican families (Matos, Torres, Santiago, Jurado, \& Rodriquez, 2006), Latino/a families (Borrego et al., 1999), separation anxiety disorder (Pincus, Eyberg, \& Choate, 2005), developmental disorders (Bagner \& Eyberg, 2007; McDiarmid \& Bagner, 2005), foster-parents (Timmer et al., 2005a, 2006), and group-therapy (Niec et al., 2005).

Utilizing Hanf's work as a template, Eyberg expanded upon this model and developed PCIT as a manualized, evidence-based treatment program to treat externalizing and internalizing behavior problems in children between 2 and 7-years-of-age (Hembree-Kigin \& McNeil, 1995; Herschell \& McNeil, 2005). During PCIT, caregivers are taught the basic theoretical components of the treatment and are then actively coached in these practices with their child. PCIT consists of two distinct phases which utilize different types of specific parenting skills. The first phase is Child Directed Interaction (CDI), which centers on building a strong and positive parent-child relationship (Bagner \& Eyberg, 2007; Hembree-Kigin \& McNeil, 1995; Herschell \& McNeil, 2005). The second phase is Parent Directed Interaction (PDI), which centers on teaching caregivers non-violent methods to decrease their child's negative behaviors while also increasing their child's positive behaviors (Bagner \& Eyberg, 2007; McNeil et al., 2001). Prior to beginning treatment, caregiver-child dyads are required to complete three tasks, outlined in the Manual for the Dyadic Parent-Child Interaction Therapy Coding System, Third Edition (DPICS-III; Eyberg, McDiarmid Nelson, Duke, \& Boggs, 2004), which allow PCIT therapists to gather baseline data 
regarding the quality of the caregiver-child relationship. In the first task, Child Directed Interaction (CDI), caregivers are advised to allow their child to lead during the play and are instructed to follow their child's lead. In the second task, Parent Directed Interaction (PDI), caregivers are instructed that they are to lead the play and they are required to engage their child in their play according to the caregiver's rules. In the final task, Clean-Up (CU), caregivers are told to have their child clean-up therapy toys and caregivers are not permitted to assist their child with this task (Eyberg, 1999). These data are gathered as part of the intake process and are utilized to assess parents' strengths and weaknesses and provide a beginning-point and guide for subsequent treatment.

CDI is designed to instruct caregivers on positive methods to utilize while interacting with their child that allow for the development of a strong caregiver-child relationship, improve caregiving skills, develop adaptive child social skills, and increase child self-esteem (Bagner \& Eyberg, 2007; Chaffin et al., 2004; Hembree-Kigin \& McNeil, 1995; Herschell \& McNeil, 2005). During this phase, caregivers are advised on how to combine play therapy skills with the concept of differential reinforcement of other behaviors (DRO) to alter their child's behaviors (Hembree-Kigin \& McNeil, 1995; Herschell \& McNeil, 2005; Timmer et al., 2005b). Caregivers are taught to deliver specialized skills (Praise, Reflection, Imitation, Description, and Enthusiasm; PRIDE Skills) to increase their child's adaptive behaviors and to reduce or eliminate certain caregiver strategies (Questions, Commands, and Criticism; QCC Skills) which remove the direction of the play from the child (Eyberg, 1999; Hembree-Kigin \& McNeil, 1995). Caregivers are coached using these skills to deliver positive verbal reinforcement to increase desired and appropriate behaviors while simultaneously ignoring disruptive behaviors to decrease their severity and frequency (Eyberg, 1999; Herschell \& McNeil, 2005). When 
caregivers have demonstrated mastery with the PRIDE skills, the second component of PCIT is initiated with caregivers (Eyberg, 1999; Niec et al., 2005).

PDI is designed to teach caregivers how to deliver clear, specific, and effective commands to their child while simultaneously utilizing the skills developed and refined during the CDI phase of treatment. In addition to learning how to deliver commands, caregivers are also taught age-appropriate expectations for their child along with numerous discipline techniques they may employ to manage their child's inappropriate behaviors and increase their child's compliance to appropriate requests (Bagner \& Eyberg, 2007; Hembree-Kigin \& McNeil, 1995; Herschell \& McNeil, 2005). These techniques include the delivery of effective commands, implementation of praise for appropriate behaviors, and consistent consequences for inappropriate behaviors (Niec et al., 2005). The major discipline technique on which caregivers receive instruction is a time-out procedure (Hembree-Kigin \& McNeil, 1995; Herschell \& McNeil, 2005). This time-out procedure involves placing a child in a specific location (usually a chair in a corner of a room) where the child is required to remain for 3-minutes. This time-out procedure is maintained until the child demonstrates obedience with the original request (Eyberg, 1999; McNeil et al., 2001).

Additionally, caregivers are taught other, non-violent discipline techniques such as distraction/redirection, reasoning, consequences, modeling, and praise/rewards to alter their child's behaviors (Eyberg, 1999). Distraction or redirection may be utilized by caregivers when a child is demonstrating inappropriate behaviors or engaging in inappropriate activities. Caregivers are provided with precise instructions on how to refocus their child's attention towards a more appropriate activity and maintain this attention on that activity. Reasoning or proving a rule for a command is also used either prior to the delivery of a command or subsequent to a child's 
compliance to a command in order for caregivers to explain the importance of their request. Consequences for inappropriate behaviors are taught as alternatives to time-out procedures in order to reduce inappropriate behaviors. Modeling is utilized by caregivers to demonstrate to children the behaviors they deem appropriate. Lastly, caregivers are taught how to praise and reward behaviors that are the opposite of inappropriate behaviors. For example, if a child often jumps on the couch, caregivers are taught to praise a child for sitting on the couch and to reward the child for that incompatible, appropriate behavior.

Caregivers with a history of CAN often perceive their children more negatively and believe their children will not respond to non-violent discipline strategies (Chaffin et al., 2004). It is often excessive discipline and inappropriate discipline strategies that result in caregivers initiating CAN towards their children (Chaffin et al., 2004). Therefore, these non-violent techniques are excellent alternatives to teach caregivers who have previously implemented CAN towards their children. In-session coaching allows the therapist to normalize certain child or caregiver behaviors and the caregiver is granted a "cooling-off" period while the child is placed in time-out. PCIT is therefore an evidence-based practice that is particularly well-suited for CAN populations due to its non-violent discipline methods which demonstrate numerous alternative ways to modify children's behaviors. Furthermore, PCIT alters caregivers who currently engage in physically violent discipline skills and teaches them more positive physical and verbal discipline skills to increase a child's appropriate behaviors and compliance to reasonable commands.

\section{Unique Components and Strengths of Parent-Child Interaction Therapy}

PCIT has many unique treatment components when compared to more traditional psychotherapies. To begin, PCIT is novel in that caregivers are actively engaged as "co- 
therapists" in their child's treatment (Eyberg, 1999; Hembree-Kigin \& McNeil, 1995; Herschell \& McNeil, 2005). Utilizing caregivers as co-therapists is useful for a variety of reasons. First, as their child's primary caregivers, they have the opportunity to spend 168-hours per week with their child compared to therapists who spend 1-hour per week with their child (Herschell, Calzada, Eyberg, \& McNeil, 2002), thereby having a far greater opportunity to positively impact their child's behaviors. Secondly, engaging caregivers as co-therapists allows them to assume responsibility for their child's behavioral and emotional changes. Lastly, caregivers gain confidence in their abilities to effect therapeutic behavior changes in their child by utilizing the skills taught during PCIT (Herschell \& McNeil, 2005).

A second component to PCIT is that both phases of treatment are first taught to caregivers using didactic instruction and modeling (Chaffin et al., 2004; Hembree-Kigin \& McNeil, 1995; Herschell \& McNeil, 2005; Chadwick, 2004). PCIT therapists model concepts to caregivers via therapist-caregiver role-playing in order to demonstrate and solidify the techniques (Chaffin et al., 2004; Hembree-Kigin \& McNeil, 1995; Herschell \& McNeil, 2005; Chadwick, 2004). This allows caregivers to practice skills and trouble-shoot potential challenges prior to implementing these skills with their child as well as increase their confidence in their ability to use these special skills.

Another original component of PCIT is that caregivers are coached and receive live feedback regarding their mastery of specific PCIT skills (Chaffin et al., 2004; Eyberg \& Robinson, 1982; Niec et al., 2005). Via a one-way mirror, therapists are able to observe parentchild interactions and coach caregivers via a "bug-in-the-ear" hearing device (Chaffin et al., 2004) which allows the therapist to provide immediate feedback to the caregivers regarding their interactions with their child (Niec et al., 2005; Timmer et al., 2005b). Conducting live, in-session 
coaching provides caregivers with precise methods to increase positive interactions with their child. These methods include how to verbally increase positive behaviors and decrease negative behaviors and numerous alternatives that caregivers may utilize to manage their child's maladaptive behaviors.

A fourth novel component to PCIT is that caregivers must demonstrate certain levels of proficiency (predetermined mastery criteria) in the CDI phase of treatment prior to being advanced to the PDI phase of treatment. Caregivers must then demonstrate certain levels of proficiency (predetermined mastery criteria) in PDI prior to successful completion of the PCIT program (Eyberg, 1999; Hembree-Kigin \& McNeil, 1995; Herschell \& McNeil, 2005). During both phases, PCIT skill mastery is assessed via a 5-minute caregiver-child observation conducted prior to each subsequent treatment session and treatment continues until caregivers demonstrate proficiency in both phases (Bagner \& Eyberg, 2007). Mastery criteria for the CDI component of PCIT require that caregivers deliver at least 10 labeled praises, 10 reflections, and 10 behavioral descriptions while simultaneously having 3 or less commands, criticisms, or questions during the same 5-minute assessment period (Eyberg, 1999; Herschell et al., 2002). Mastery criteria for the PDI component of PCIT require that caregivers deliver a minimum of four effective commands (i.e., clear, direct, positively declared, and with an opportunity for the child to comply) with a minimum of $75 \%$ (child and caregiver) proper follow-through subsequent to the original command (i.e., opportunity for child compliance, warning for non-compliance, labeled praise for compliance) (Eyberg, 1999; Herschell et al., 2002; Werba, Eyberg, Boggs, \& Algina, 2006).

Lastly, PCIT is unique in its ability to generalize treatment gains made in the clinic setting to alternative environments. During standard therapy, once a child's maladaptive behaviors have been eliminated, treatment is terminated. However, these maladaptive behaviors 
may reappear at a later time or may manifest in different forms of maladaptive behaviors. PCIT teaches parents skills that they can use in the future to alter their child's behaviors as necessary. This is accomplished through the use of "homework" which caregivers are asked to complete with their child, at home, for a minimum of 5-minutes per day (Bagner \& Eyberg, 2007). This allows for PCIT skills which are developed in-session to "spill-over" or generalize to multiple environments, ensuring that children know that their caregivers' rules and expectations are consistent (Chadwick, 2004; Hembree-Kigin \& McNeil, 1995).

These innovative approaches to treatment have demonstrated success with a variety of populations and have been adapted to meet each of these populations' specific needs (Eyberg, 2005). While PCIT may have novel approaches to treatment, this therapy consists of adaptations that may benefit children and families affected by CAN. Historically, conventional psychotherapy has focused on treating one client (either the parent or child) individually; alternatively PCIT treats both caregivers and children simultaneously (Bell \& Eyberg, 2002; Borrego et al., 1999). To begin, both caregivers who inflict CAN and children who are the recipients of CAN take active roles in the therapeutic process (Chaffin et al., 2004; Timmer et al., 2005b). Standard PCIT typically focuses on altering children's maladaptive behaviors. However, when working with caregivers who have inflicted CAN, the primary treatment goal shifts towards altering the caregiver's maladaptive behaviors (Borrego et al., 1999).

\section{Criticisms of Parent-Child Interaction Therapy}

Manualized-based treatment programs have been accused of being incompatible with a behavioral approach to therapy (Greco, Sorrell, \& McNeil, 2001). Specifically, these treatment programs have been accused of being "cookbook" methods of treatment that do not consider individual differences in the conceptualization, planning, and delivery of individualized 
treatments (Greco et al., 2001). One criticism of PCIT is that it is a nomothetic treatment which does not consider or address individual specific treatment needs. In actuality, PCIT is both a nomothetic treatment and an idiographic treatment. PCIT is nomothetic in nature in that it is a manualized treatment with very structured session outlines. Treatment usually consists of approximately 14 sessions (6 CDI, 6 PDI, 2 Didactic) and caregivers are taught predetermined skill sets in a sequence that research has demonstrated to be most effective (Hembree-Eisenstadt, Eyberg, McNeil, Newcomb, \& Funderburk, 1993). Another nomothetic component of PCIT is its utilization of norm-based assessment measures to monitor child behavior changes. However, many treatments (nomothetic or idiographic) utilize standardized measures to assess treatment progress.

Alternatively, PCIT is also idiographic in nature for a multitude of reasons. First, while PCIT has two pre-ordained phases of therapies, the quantity of treatment sessions is actually flexible based upon a family's strengths and weakness and successful progression through treatment. For example, fewer sessions may be required if the family demonstrates mastery of the skills quickly and more sessions may be added if necessary for the family to further develop pertinent skills. Second, while there are pre-determined mastery criteria for PCIT, each caregiver's progress is individually monitored and sessions are customized to address the specific needs of caregivers and children (Hembree-Kigin \& McNeil, 1995; Herschell \& McNeil, 2005). Third, while PCIT does utilize norm-based assessment measures to monitor treatment progress, these assessment measures are also utilized in an idiographic fashion. For example, information derived from these measures may assist in the development of individualized treatment plans for clients and individualized feedback for the parents (McNeil et al., 2001). These assessment measures also allow for the targeting of specific behaviors, a guide for 
treatment, a means to evaluate treatment progress, and an instrument to engage caregivers in treatment (Niec et al., 2005). Lastly, PCIT therapists work diligently to establish rapport with families and alter their coaching style to most closely match the individual needs of the child and family in treatment (Herschell et al., 2002).

\section{Parent-Child Interaction Therapy and Child Abuse and Neglect Populations}

Many therapies target only the caregiver or child during treatment. PCIT is unique as it incorporates both the caregiver and child in treatment in order to address and alter deficiencies in the caregiver-child relationship (Borrego et al., 1999; Ware, Fortson, \& McNeil, 2003). In truth, it is actually the caregivers who are the main focus in treatment for initiating CAN towards their child (Borrego et al., 1999; Brinkmeyer \& Eyberg, 2003). The primary goal of PCIT is to target and alter the caregiver-child interaction patterns to be more positive and reinforcing to both parties (Borrego, et al., 1999; Ware et al., 2003). Research conducted by Timmer and colleagues (2002) has demonstrated that caregivers who do not have a history of CAN provide their children with higher levels of verbal praises, initiate higher levels of positive behaviors and lower levels of negative behaviors towards their children, and engage in more total verbalizations with their children compared to caregivers with a history of CAN. Furthermore, children in this study demonstrated similar behavioral patterns as their caregivers. Children without a history of CAN demonstrated higher levels of verbal praises, initiate higher levels of positive behaviors and lower levels of negative behaviors towards their caregiver, and engaged in more total verbalizations with their caregiver compared to children with a history of CAN. PCIT is designed to target the negative interactions that occur within the parent-child dyad and to enhance the relationship by teaching caregivers how to interact positively with their child. 
Borrego and colleagues (1999) conducted the first study examining the effects of PCIT with a child who had experienced CAN implemented by his biological mother. The authors employed a case-study design with a 35-year-old single mother and her 3-year-old son who also suffered from fetal alcohol syndrome. Results indicated that the potential for CAN (as indicated by the Child Abuse Potential Inventory (CAP; Milner, 1986)) and parental stress (as indicated by the Parenting Stress Index (PSI; Abidin, 1995)) decreased from pre-treatment to 16-month follow-up. In addition, positive parental verbal interactions (as indicated by verbal descriptions and praises) increased from pre- to post-treatment and negative parental verbal interactions (questions) decreased from pre- to post-treatment. Furthermore, the number and intensity of child behavior problems (as indicated by the Eyberg Child Behavior Inventory (ECBI; Eyberg \& Pincus, 1999)) and child internalizing and externalizing behavior problems (as indicated by the Child Behavior Checklist (CBCL; Achenbach, 2001; Achenbach \& Rescorla, 2000)) decreased from pre-treatment to 16-month follow-up. These results indicate that PCIT is effective in treating parents who implement CAN towards their child by reducing future CAN potential, decreasing parental stress, decreasing child behavior problems, and increasing positive parentchild interactions.

Chaffin and colleagues (2004) utilized PCIT in a randomized trial with physically abusive parents and their children. Participants included 110 parents with at least one child who had experienced physical abuse between 2 and 12-years-of-age. Participants were distributed into one of three groups: (a) standard PCIT, (b) PCIT plus individualized enhanced services, and (c) standard community services. Data were gathered regarding recidivism rates for child physical abuse approximately 850 days after treatment. These data provided evidence regarding the three different forms of treatment. It was found that 37 (34\%) of the 110 participants committed child 
physical abuse following treatment. Eight (19\%) of these participants were from the PCIT only treatment, $12(26 \%)$ were from the PCIT plus individualized enhanced services, and 17 (49\%) received standard community services. This study provides evidence that PCIT is an effective form of treatment for child physical abuse.

Timmer and colleagues (2005b) conducted an effectiveness study of PCIT with children who displayed externalizing behavior problems and either had experienced CAN or had not experienced CAN. Participants included 136 children (91 children with a history of CAN, 45 children without a history of CAN) between 2 and 8 years-of-age and at least one biological caregiver. Regardless of maltreatment history, children in both groups (maltreated and nonmaltreated) reported overall decreases in internalizing, externalizing, and total behavior problems, via the CBCL (Achenbach, 2001; Achenbach \& Rescorla, 2000), and fewer problem behaviors with less intensity, via the ECBI (Eyberg \& Pincus, 1999). Furthermore, biological caregivers in both groups (maltreated and non-maltreated) also demonstrated decreases in parental stress, parental psychopathology, and CAN from pre-to post-treatment for both treatment groups.

Dombrowski and colleagues (2005) conducted a case-study utilizing a modified version of PCIT, entitled Parent-Child Attunement Therapy (PCAT), which was developed for children 12 to 30-months-of-age. PCAT was utilized to treat a 23-month-old male with a history of maltreatment and his biological mother. His biological mother gave birth to him while she was incarcerated and the child may have had prenatal drug exposure. He was placed in foster care until he was 22-months-of-age when his biological mother resumed custody. At this time he began exhibiting aggressive behaviors towards himself and others. While pre- and post-treatment behaviors on his CBCL (Achenbach, 2001; Achenbach \& Rescorla, 2000) and ECBI (Eyberg \& 
Pincus, 1999) and his mothers PSI (Abidin, 1995) did not demonstrate clinically significant improvements, positive changes in the quality of the parent-child relationship were present at post-treatment.

Timmer and colleagues (2005a) also utilized PCIT in a single-case study design with a 41-year-old married foster mother and her 4-year-old foster son. At 16-months of age, the son had been removed from his mother's custody when his newborn sibling tested positive for illegal drugs. Since that time he had been placed with six other foster placements prior to his current placement. During one of those placements, he was removed from his foster parents care when they were accused of physical abuse towards another child. His mother attempted reconciliation but then abruptly terminated her parental rights. PCIT was initially begun with his biological mother to treat the boy's self-injurious behaviors and sexualized behaviors. However, upon termination of parental rights, PCIT was continued with his new foster mother. During treatment with his foster mother, the potential for CAN (as indicated by the CAP (Milner, 1986)) decreased from pre- to post-treatment and parental stress (as indicated by the PSI (Abidin, 1995)) decreased from pre- to post-treatment. In addition, positive parental verbal interactions (as indicated by verbal descriptions and praises) increased from pre- to post-treatment and negative parental verbal interactions (questions) decreased from pre- to post-treatment. Furthermore, the number and intensity of the child's behavior problems (as indicated by the ECBI (Eyberg \& Pincus, 1999)) decreased from pre- to post-treatment and child internalizing, externalizing, and total problem scores (as indicated by the CBCL (Achenbach 2001; Achenbach \& Rescorla, 2000)) decreased from pre- to post-treatment. These results indicate that PCIT is effective in treating children with a history of CAN who are currently living with a non-abusive caregiver. 
Timmer and colleagues (2006) conducted a study examining the effectiveness of PCIT with biological- and foster-caregivers and children with a history of CAN. Participants included 98 non-abusive biological caregiver-child dyads and 75 non-abusive foster-care caregiver-child dyads. Regardless of caregiver status (biological versus foster care) PCIT was effective for reducing the intensity and number of problem behaviors, internalizing, externalizing, and total behavior problems, caregiver psychopathology, caregiver stress, and the potential for future child abuse. This study was able to demonstrate the effectiveness of PCIT as a potential treatment for CAN in a community setting.

In conclusion, PCIT has received support as an evidence based treatment for CAN and encouraging results have been documented in several studies (Borrego et al., 1999; Chaffin et al., 2004; Dombrowski et al., 2005; Hershell \& McNeil, 2005; Timmer et al., 2005a; Timmer et al., 2005b; Timmer et al., 2006; Ware et al., 2003). Data from these studies have demonstrated that PCIT is effective in reducing CAN recidivism rates (Chaffin et al., 2004; Timmer et al., 2005a; Timmer et al., 2006), decreasing parental stress (Borrego et al., 1999; Timmer et al., 2005a; Timmer et al., 2005b; Timmer et al., 2006), decreasing parental psychopathology (Timmer et al., 2005a; Timmer et al., 2006), decreasing the number of child behavior problems (Borrego et al., 1999; Timmer et al., 2005b; Timmer et al., 2006), decreasing child internalizing behaviors (Borrego et al., 1999; Timmer et al., 2005a; Timmer et al., 2005b; Timmer et al., 2006), decreasing child externalizing behaviors (Borrego et al., 1999; Timmer et al., 2005a; Timmer et al., 2005b; Timmer et al., 2006), decreasing total behavior problems (Timmer et al., 2005a; Timmer et al., 2005b; Timmer et al., 2006), and improved parent-child relationship (Dombrowski et al., 2006). 
Parent Child Interaction Therapy 22

Parent-Child Interaction Therapy and Intimate Partner Violence

Although PCIT has received theoretical support in the literature as a potential treatment for IPV (Borrego, Gutow, Reicher, \& Barker, 2008; Pearl, 2008), to date, no research has been conducted that has assessed the impact of PCIT upon IPV. Based upon case studies that have treated children who have experienced CAN and also have been exposed to IPV, research has elucidated that PCIT skills that caregivers learn to utilize with their children may generalize to their relationships with others. Eyberg and Robinson (1982) conducted a study examining how PCIT skills improved family functioning in preschool-aged children. Their results also demonstrated that PCIT skills generalized to untreated siblings and resulted in the decrease of maladaptive behavior, the decrease of non-compliance, and the increase in compliance for these untreated siblings. Brestan and colleagues (1997) were able to replicate these findings utilizing a randomized control trial study design. Their results indicated that maternal and paternal caregivers in the immediate treatment group reported a greater decrease in untreated siblings' behavioral problems compared to untreated siblings in the waitlist control group. Caregivers in these two studies had learned to implement PCIT techniques with a target child enrolled in the study, but they had also begun to use these techniques with their other children. As a result, these untreated siblings also demonstrated improvements in behavior and functioning.

PCIT may also be an effective treatment for IPV due to its conjoint approach to treatment, short duration, and highly structured course of treatment. Many treatments for female and male partners involved in IPV relationships consists of separate therapy sessions for females and males with same-gender therapists (Harris, 2006; O’Leary, 2002). However, research has shown that this may not be the most effective form of treatment and that conjoint therapy may be more effective at reducing IPV recidivism (Harris, 2006; O’Leary, 2002). PCIT involves both 
caregivers in treatment and focuses on teaching both caregivers the same PCIT skills to utilize with their child. As both caregivers are utilizing the same skills set, and PCIT skills have been shown to generalize to other family members and improve functioning, it is plausible that caregivers who experience IPV may also begin using these PCIT skills within their own relationships. The skills taught during PCIT focus on increased warmth and communication within a relationship and also teach non-violent methods in which to gain compliance to reasonable commands. These skills have been shown to decrease CAN and may also generalize to decrease IPV.

Additionally, research has demonstrated that treatments targeting IPV that were shorter in length and were more structured were more effective at reducing IPV recidivism. Edleson and Syers (1990) conducted a study examining the effectiveness of six different types of group treatment for males who implemented IPV towards their female partners. Results indicated that treatments that were shorter in duration (12-sessions versus 32-sessions) and were more structured (psychosocial education versus self-help classes versus combination) were more effective at reducing IPV recidivism. Specifically, males enrolled in a 12-session group had 10\% lower IPV recidivism rates compared to males enrolled in a 32-session group, regardless of group assignment. Furthermore, males from the more structured psychosocial education group had $32.3 \%$ IPV recidivism rates compared to males form the self-help group who had 53.8\% IPV recidivism rates. These results suggest that PCIT may prove to be a successful treatment for IPV for three reasons: (a) it is a time-limited approach, (b) it focuses on applying skills in session, and (c) it works with the couple conjointly focusing on using a consistent and positive approach to all relationships. 
Indeed, as the separate research fields of CAN and IPV have advanced, it appears that these two fields were examining a broader concept of family violence (co-occurring CAN and IPV) that has emerged as a field in its own right (Appel \& Holden, 1998). Since CAN and IPV have such a high co-morbidity rate, it is sensible to develop a treatment that addresses both forms of family violence. Research has indicated that PCIT may be utilized to treat CAN; however, there may be secondary gains in that it concurrently may also treat components of IPV. Hence, PCIT may serve as a "two-for-one" treatment in its ability to potentially reduce two forms of family violence. PCIT has been used successfully in clinical settings with families with a history of IPV in reducing maladaptive child behaviors (Herschell \& McNeil, 2005). However, no study to date has evaluated the effects of PCIT on reducing IPV and its effects upon women, children, and families. A study that assessed PCIT's effects upon IPV would provide valuable information regarding treatment outcome (Herschell \& McNeil, 2005).

\section{Statement of the Problem}

Child abuse and neglect (CAN) and intimate partner violence (IPV) are serious public health issues that need to be addressed. In 2005, a reported 899,000 children throughout the United States, the District of Columbia, and Puerto Rico were the victims of either abuse or neglect resulting in the death of 1,460 children under 18-years-of-age (US Department of Health and Human Services, 2005). In the United States each year, adult women are subjected to over 5 million IPV incidences and which result in the death of approximately 1,300 women (APA, 2005). A meta-analysis of 31 studies conducted by Appel and Holden (1998) examining the cooccurrence of IPV and CAN revealed that these two forms of violence range from $20 \%-100 \%$ co-morbidity with an average of $41 \%$ co-morbidity. PCIT is an evidence-based treatment that has both theoretical and scientific support as a treatment to reduce future CAN recidivism and 
decrease the short- and long-term effects of CAN on children (Borrego et al., 1999; Chaffin et al., 2004; Timmer et al., 2005a; Timmer et al., 2005b, Timmer et al., 2006). Although PCIT has received theoretical support in the literature as a potential treatment for IPV (Borrego, Gutow, Reicher, \& Barker, 2008; Pearl, 2008), to date, no research has been conducted that has assessed the impact of PCIT upon IPV. Currently, no studies have been conducted to examine the effectiveness of PCIT in a community-based environment with co-morbid CAN and IPV populations. This study aimed to contribute to the fields of CAN, IPV, and PCIT by evaluating the effectiveness of PCIT with populations who experience co-morbid family violence.

The current study was designed to fill several voids in the literature. As a result, this is the first study in the literature to address the following issues:

1. Evaluating whether PCIT decreases child abuse potential when conducted in a community setting using a group format, above and beyond treatment as usual.

2. Evaluating whether PCIT decreases IPV in the caregivers, above and beyond treatment as usual.

3. Evaluating whether PCIT decreases behavior problems in children exposed to IPV and child physical abuse more than treatment as usual.

4. Evaluating whether group PCIT can be effective in the community (without specialized equipment or laboratory space) with CAN families in terms of decreasing parental stress, decreasing child behavior problems, and increasing parental skill acquisition.

To address these concerns, the current study uses a two group design, comparing PCIT and treatment as usual at pre- and post-treatment. Measures were designed to evaluate traditional PCIT outcomes (e.g., child behavior, parenting stress, parenting skill), as well as the additional domains of caregiver IPV and child abuse potential. 
Hypotheses

Hypothesis 1. Child Abuse and Neglect. Potential child abuse and neglect risk as indicated by the Child Abuse Potential Inventory (CAP; Milner, 1986) would be related to treatment condition. It was hypothesized that the CAP would decrease more in the Parent-Child Interaction Therapy (PCIT) condition compared to the treatment as usual (TAU) condition from pre- to post-treatment.

Hypothesis 2. Intimate Partner Violence. Intimate partner violence as indicated by the Revised Conflict Tactics Scale (CTS2; Straus, Hamby, Boney-McCoy, \& Sugarman, 1996) would be related to treatment condition.

Hypothesis $2 a$. It was hypothesized that the male-to-female psychological violence would decrease more in the PCIT condition compared to the TAU condition from pre- to posttreatment.

Hypothesis $2 b$. It was hypothesized that the female-to-male psychological violence would decrease more in the PCIT condition compared to the TAU condition from pre- to posttreatment.

Hypothesis $2 c$. It was hypothesized that the male-to-female physical violence would decrease more in the PCIT condition compared to the TAU condition from pre- to posttreatment.

Hypothesis $2 d$. It was hypothesized that the female-to-male physical violence would decrease more in the PCIT condition compared to the TAU condition from pre- to posttreatment.

Hypothesis 3. Parental Stress. Parental stress as indicated by the Parenting Stress IndexShort Form (PSI-SF; Abidin, 1995) would be related to treatment condition. It was hypothesized 
that the PSI-SF total stress would decrease more in the PCIT condition compared to the TAU condition from pre- to post-treatment.

Hypothesis 4. Child Behavior. Child behavior as indicated by the Child Behavior Checklist (CBCL; Achenbach, 2001; Achenbach \& Rescola, 2000) and Dyadic Parent-Child Interaction Coding System-III (DPICS-III; Eyberg, McDiarmid Nelson, Duke, \& Boggs, 2004) Child Compliance category would be related to treatment condition.

Hypothesis 4a. It was hypothesized that the internalizing behavior problem scores on the CBCL would decrease more in the PCIT condition compared to the TAU condition from pre- to post-treatment.

Hypothesis $4 b$. It was hypothesized that the externalizing behavior problem scores on the CBCL would decrease more in the PCIT condition compared to the TAU condition from pre- to post-treatment.

Hypothesis 4c. It was hypothesized that the total behavior problem scores on the CBCL would decrease more in the PCIT condition compared to the TAU condition from pre- to posttreatment.

Hypothesis $4 d$. It was hypothesized that the DPICS-III Child Compliance score would increase in the PCIT condition from pre- to post-treatment.

Hypothesis $4 e$. It was hypothesized that ECBI Intensity score would decrease more in the PCIT condition compared to the TAU condition from pre- to post-treatment.

Hypothesis $4 f$. It was hypothesized that ECBI Problem score would decreased more in the PCIT condition compared to the TAU condition from pre- to post-treatment. 
Hypothesis 5. Parenting Behavior. Parenting behavior as indicated by the DPICS-III Positive Talk would increase and Negative Talk would decrease in the PCIT treatment condition from pre- to post-treatment.

Hypothesis 5a. It was hypothesized that DPICS-III Positive Talk scores would increase in the PCIT condition from pre- to post-treatment.

Hypothesis $5 b$. It was hypothesized that DPICS-III Negative Talk scores would decrease in the PCIT condition from pre- to post-treatment.

\section{Method}

\section{Participants}

Participants were recruited from The Family Junction, Inc., a community outreach organization located in Cumberland, Maryland. Cumberland, MD is a small city (population: 25,000) located in Allegheny County; approximately 130 miles west of Washington, DC. The Family Junction, Inc., serves approximately 50-70 families per year and primarily offers parenting classes to: (a) families affected by child abuse, and/or child neglect, (b) families at risk of child abuse or neglect, and (c) families with substantiated CAN reports.

Forty-nine caregiver-child dyads were recruited to participate in this study. Inclusionary criteria for participation in this study included: (a) the child's family was referred (or selfreferred) to The Family Junction, Inc. for parenting services related to either a documented history of child physical abuse and/or neglect or a perceived risk for child physical abuse or neglect, (b) the caregiver's family included a target child 2 to 12-years-of-age who may or may not have been residing in the home at the point of referral, and (c) the target child (2 to 12-yearsof-age) was available to be observed with the caregiver during pre- and post-treatment evaluations. Exclusionary criteria for participation in this study included: (a) a caregiver under 
18-years-of-age, (b) the caregiver did not speak English fluently, and (c) child(ren) were referred exclusively or primarily for issues related to child sexual abuse. No children were referred for issues related to child sexual abuse.

\section{Procedure}

The Institutional Review Board at West Virginia University reviewed and approved the study protocol and informed consent forms prior to participant recruitment. Recruitment occurred between August 2008 and December 2009 with data collection ending in March 2010. As a component of the intake procedure at The Family Junction, Inc., eligible caregivers were approached to participate in a research study examining the effectiveness of PCIT services and treatment as usual services. Potential participants who declined to participate in this research project were offered treatment as usual services at The Family Junction, Inc. and no data was gathered from these families. Only one family declined to participate in this study. The research project was explained to participants by research staff members, consent was read to participants and obtained prior to completing pre-treatment questionnaires, and participants were provided with a copy of their consent form. Assistance was provided by research staff members if necessary to assist participants in completing questionnaires. All participants were assigned an identification number and all assessment measures were labeled with this identification number in order to ensure privacy of responses.

Upon enrollment, participants were assigned to one of two treatment conditions: (a) Parent-Child Interaction Therapy, or (b) treatment as usual. Group assignment was not random and participants were assigned to conditions based upon which group was able to accept participants at that time. The Parent-Child Interaction Therapy (PCIT) condition consisted of 21 participants who received weekly, two-hour, group PCIT sessions over the course of 12-weeks. 
The PCIT treatment groups were comprised of approximately 4-10 caregiver-child dyads and were lead by two co-therapists in order to ensure quality control (Chaffin et al., 2004). The PCIT condition received six 6-CDI sessions and 6-PDI sessions (12-sessions total). Of note, there was slight contamination in the PCIT condition as two caregivers were previously married. Each caregiver was court-ordered to attend treatment and it was determined that it was appropriate to include the caregivers in the same program to promote consistency regarding parenting methods. Due to interpersonal conflict, each caregiver was included in a different PCIT group and assessment measures were gathered on two different children.

The treatment as usual (TAU) condition consisted of 28 participants who received weekly, two-hour, group sessions (as developed by The Family Junction, Inc.) over the course of 12-weeks. The TAU groups were comprised of up to 18 caregivers and were lead by two or three therapists in order to ensure quality control (Chaffin et al., 2004). The inclusion of up to 18 caregivers was permitted in order to maintain standard TAU treatment integrity as groups at The Family Junction, Inc. have averaged between 12-20 caregivers over the past six years. The TAU protocol development is best understood by examining the history of the agency. Approximately 13 years ago, an assessment conducted in Cumberland, MD determined that parenting was ranked as one of the community's top needs. Grant funding allowed for the development of The Family Junction, Inc. which filled this need by offering a curriculum that focused on delivering psychosocial education on topics such as time and stress management, long-range parenting, communication, discipline, and natural and logical consequences. The services offered by this organization were developed based upon other parenting programs, educational resources, and information learned from conferences focusing on the delivery of parenting services in community-based organizations. Since its inception, the curriculum at The Family Junction, Inc. 
has been revised three times and the current protocol (TAU) has been utilized for approximately two years.

Participants were informed they could withdraw from the research project at any time without penalty. No participants formally withdrew from this study but several did discontinue treatment services with The Family Junction, Inc. Participants were compensated for participating in this research project and completing measures for one child. Caregivers completed a battery of assessment measures administered at two time-points throughout the duration of the study. Specifically, assessment measures were administered at pre- and posttreatment. Pre-treatment measures were presented in the following order: Demographics Form, Child Behavior Checklist, Revised Conflict Tactics Scale, Brief Symptom Inventory, Child Abuse Potential Inventory, Parenting Stress Index-Short Form, Eyberg Child Behavior Checklist, and Dyadic Parent-Child Interaction Coding System-III and were completed by participants in approximately 65 to 80 -minutes. Post-treatment measures were presented in the following order: Child Behavior Checklist, Revised Conflict Tactics Scale, Child Abuse Potential Inventory, Parenting Stress Index-Short Form, Eyberg Child Behavior Checklist, Dyadic Parent-Child Interaction Coding System-III, and Therapy Attitude Inventory and were completed by participants in approximately 57 to 70 -minutes.

Participants in both treatment conditions were reimbursed $\$ 30.00$ for completing pretreatment assessment measures and $\$ 70.00$ for completing post-treatment assessment measures (total: $\$ 100.00)$ as a way of compensating them for their time-investment. Participants were offered a larger financial reimbursement at post-treatment as an effort to gather post-treatment data from participants who did not complete treatment so that their data could be used to analyze their outcomes. Research has indicated that attrition rates for child treatment programs range 
between 40 to $60 \%$ (Wierzbicki \& Pekarik, 1993) and it was expected that attrition rates for this research study would be similar. In order to combat attrition across all research participants, several methods were utilized: (a) participants were asked to sign an attendance contract prior to beginning treatment which specifically stated that they were required to attend all scheduled sessions and to contact research staff in the event they were unable to attend a session, (b) participants received a telephone call the day prior to their scheduled treatment session which served as a reminder of their appointment, what materials they needed to bring to that session, and allowed for the coordination of transportation if necessary, and (c) families that occasionally did not attend sessions were provided with an opportunity to attend a group or individual makeup session for that treatment session. In addition to financial compensation, all participants received psychotherapy services free of charge, complimentary meals and healthy snacks during treatment sessions, free transportation to and from treatment sessions (if necessary), complimentary child care during sessions, and toy kits to keep at the completion of treatment. There was a $9.5 \%$ attrition rate for the PCIT condition $(\mathrm{N}=2)$ and a $14.5 \%$ attrition rate for the TAU condition $(\mathrm{N}=4)$. Participants in the PCIT condition attended on average 8.10 classes $(S D$ $=3.18)$ compared to those in the TAU condition who attended 6.11 classes $(S D=3.00)$. See Table 1.

\section{Parent-Child Interaction Therapy Condition}

Treatment followed the standard PCIT protocol (Eyberg, 1999) with the exception that treatments were structured as a group format compared to an individual format. As these sessions were conducted in a group format, several modifications were necessary. One modification was that all families received a pre-determined number of treatment sessions (12 in total) in order to match the number of TAU sessions. A second modification was that participant's advancement 
from CDI to PDI and successful completion of the PCIT program was not based upon mastery criteria. In standard individual PCIT treatment, there are a pre-determined number of sessions and the number of sessions is determined based upon the caregiver's and child's ability to demonstrate mastery of PCIT skills. However, within a group format all participants must move forward at the same time, regardless of skill mastery (Niec et al., 2005). In the PCIT condition, 7 of the 21 participants (33\%) achieved PCIT skills mastery within the 12 week program. A third modification excluded the use of a time-out room for children who refused to remain in the timeout chair. Instead, children who refused to remain in the time-out chair were continuously brought back to the chair until they remained for the duration of their time-out. A fourth modification included the use of in-room, direct observation coding and coaching of PCIT skills. Lastly, a fifth modification included the use of a blanket to delineate for children the parameters of their ability to move about the facility during PCIT coding and coaching.

Each PCIT session was conducted in two-hour time periods. The first 30-minutes of each session consisted of PCIT group members having a family meal and PCIT therapists working with the families to ensure that PCIT skills were generalizing to alternative environments. During this time, caregivers, target children, and siblings were able to engage in a family dinnertime meal and caregivers were able to practice their PCIT skills to reinforce appropriate behaviors and punish inappropriate behaviors under the guidance of a trained therapist. Caregivers received immediate feedback regarding skills generalization and the time-out procedure for non-compliance was implemented under natural circumstances.

The next 75-minutes of each session consisted of assessing caregiver attendance, homework review, overview of PCIT session, PCIT mastery coding, and live, in-session coaching of individual PCIT group members. For caregivers to receive a positive letter to the 
court, they were required to attend sessions regularly and to schedule make-up sessions prior to the next group treatment session. Next, family PCIT homework from the previous week was reviewed and documented. As a component of therapy, caregivers were required to practice the PCIT skills that they learned in-session with their child at home or during supervised visitations. The expectation was that this "homework" would allow the caregiver to practice their PCIT skills on a daily basis and that these skills would generalize to environments outside of the clinic environment. Next, caregivers received instruction regarding the current PCIT lesson. Therapistcaregiver role-playing was utilized as required to demonstrate specific skills prior to coding or coaching for that session. Caregivers then completed the Eyberg Child Behavior Inventory (ECBI) and their mastery of PCIT skills were coded for 5-minutes utilizing the Dyadic ParentChild Interaction Coding System-III. Following this coding session, each caregiver, individually, was coached for a minimum of 5-minutes on skills which they needed to further develop in order to attain PCIT skill mastery. Therapeutic toys were provided during the DPICS-III coding and coaching time and accommodations for older children, such as the inclusion of age-appropriate activities, were included. During this time, PCIT participants received live, in-room feedback regarding skills acquisition and improving skills deficits. Simultaneously, other caregivers enrolled in the PCIT treatment condition were able to observe and participate in the coding and coaching process of other PCIT participants. The majority of caregivers participated in coding, coaching, and providing psychosocial support to other caregivers during this process. Verbal reports from caregivers indicated that they found this component to be extremely supportive; perhaps due to the perception that other caregivers experienced similar child behavior difficulties and were not judgmental regarding others caregiving practices. Furthermore, PCIT participants were provided with coding sheets and were taught to code other PCIT participants on their PCIT 
mastery skills. For some sessions, participants whose children were not in attendance were coded and coached with a child who was the same gender and age of their child. Involvement in this process assisted PCIT participants to better learn, understand, apply, and master these therapy skills.

The last 15-minutes of each session consisted of a review of that week's treatment plan followed by a wrap-up of the day's session. First, PCIT participants received a review of the current day's treatment session. Next, participants received feedback regarding their individual performance; specifically, which skills they had successfully mastered and which skills they need to continue to develop. Lastly, homework was disseminated for the upcoming week.

\section{Modifications from Individualized PCIT Format vs. Group PCIT Format}

The structure of individualized PCIT treatments consists of an initial intake assessment, a CDI didactic followed by CDI in-session coaching, a PDI didactic followed by PDI in-session coaching, post-treatment review/assessment, and a booster session at 1-month post-treatment (Niec et al., 2005). However, modifications are necessary when converting individualized-PCIT to a group-PCIT format. For instance, the size of PCIT groups is important to consider. While other group treatments may include up to 15 families, due to the extensive time requirement of in-session coaching, Niec and colleagues (2005) have limited PCIT groups to 6 families or less. Another consideration is time. Individualized PCIT sessions are typically one-hour in length. However, in order to coach group PCIT sessions, time must be extended to approximately $1 \frac{1 / 2}{2}$ to 2-hours dependent upon the size of the group (Niec et al., 2005). Thirdly, size constraints of the clinic and staffing requirements must also be considered when determining the appropriate size of the PCIT group. It is important to limit the size of PCIT groups based upon the number of families that can be accommodated by the space available (Niec et al., 2005). Fourthly, it is 
important to consider how many therapists will be available to deliver PCIT services. Lastly, a therapist and co-therapist typically administer PCIT to individual families (Niec et al., 2005). However, more therapists may be required for a PCIT group format dependent upon the size of the group. Furthermore, additional support staff members may be required to care for children in treatment and siblings during group sessions.

There are both advantages and disadvantages to conducting PCIT in a group format compared to an individual format. One disadvantage is that families receive less one-on-one therapist coaching time. Typically, a PCIT client would have approximately 20 to 40 minutes of individual coaching. With a two-hour group format families are allocated less direct time with a therapist. Another disadvantage is that treatment is less individualized. In a group format, all participants must progress through treatments at the same rate, regardless of skill mastery. Alternatively, an advantage of PCIT in a group format is that this service is more economical for both clients and the healthcare system while still maintaining high-quality services (Greco et al., 2001; Niec et al., 2005). Another advantage is that caregivers in PCIT groups are able to offer personal support to other caregivers in the group. Furthermore, group members are exposed to other children's behaviors which "normalize" these behaviors to caregivers. Lastly, while PCIT in a group format is less individualized, weekly idiographic assessments allow for in-session coaching addressing group member's personal strengths and weaknesses.

\section{Treatment as Usual Condition}

Each TAU session was conducted in two-hour time-periods. The first 45-minutes of each session consisted of TAU group members having a meal with their children and other TAU families. The next 60-minutes of each session consisted of two therapists leading TAU group members in a discussion regarding the day's selected topic and various methods for applying this 
topic to their lives. These topics included time and stress management, long-range parenting, relationship-building techniques, communication-enhancement techniques, rule development and behavioral limit setting, discipline techniques, and natural and logical consequences. The last 15minutes of each session consisted of a review of that week's treatment plan followed by a wrapup of the day's session. During this time, TAU condition participants received a review of the current day's treatment session. Participants also received feedback regarding their individual performance; specifically, which skills they had successfully mastered and which skills they needed to continue to develop.

\section{Treatment Fidelity and Quality Assurance}

Approximately two graduate students and five undergraduate students from West Virginia University served as therapists in the study. Both graduate students contributed equally across both PCIT and TAU conditions. The primary therapist for the PCIT condition had previous PCIT experience (i.e., participated on a clinical team specializing in PCIT for one year and conducted PCIT with over five parent-child dyads) and adhered to a detailed PCIT manual (Eyberg, 1999) which provided content to be covered during each session. Furthermore, therapists attended two, one-week, 40-hour PCIT training courses prior to beginning the research study. The primary therapist for the TAU condition has previous experience with this program (i.e., developed protocol and administered TAU for the previous six years) and adhered to a TAU outline which provided content to be covered during each session.

\section{Treatment Integrity}

Therapists received weekly, one-hour, group supervision throughout the duration of the study from a licensed clinical psychologist who possessed experience in both providing and supervising PCIT. These meetings were instrumental in maintaining treatment integrity (Nixon, 
Sweeney, \& Erickson, 2004). During supervision, therapists reviewed and discussed PCIT and TAU session content and received instruction regarding how to improve therapy and ensure treatment integrity. Furthermore, a graduate student or advanced undergraduate student assessed integrity for PCIT and TAU utilizing session-by-session protocol adherence checklists (Bagner \& Eyberg, 2007; Borrego et al., 1999; Chaffin et al., 2004; Schuhmann, Foote, Eyberg, Boggs, \& Algina, 1998). Integrity percentages were determined for each treatment session by dividing the number of session components accurately completed by the total number of session components that could be accurately completed and multiplying that number by 100 . It was determined that if integrity dropped below $80 \%$ for a session, therapists would meet with their supervisor to discuss the integrity problem and develop a corrective plan. This was not required and PCIT treatment integrity was calculated at $94 \%$ across all PCIT sessions. TAU treatment integrity was calculated at $100 \%$ across all TAU sessions.

Measures

Demographic Form (Hollingshead, Unpublished Manuscript). (Appendix A).

Demographic information gathered included: maternal age, paternal age, child age, child gender, sibling ages and genders, maternal educational attainment, paternal educational attainment, maternal ethnicity, paternal ethnicity, child ethnicity, maternal relationship status, maternal employment, paternal employment, and family income. Additional information gathered was session attendance, homework completed, participation in sessions, and whether the target child attended the session. Socioeconomic status was assessed using a Two-Factor Hollingshead Index.

Child Behavior Checklist (CBCL; 1 1/2 - 5, Achenbach \& Rescorla, 2000; 6-18, Achenbach, 2001). (Appendix B and Appendix C). The CBCL is an approximately 100-item 
measure designed to assess children's social, behavioral, and emotional problems within the previous six months. Eight narrow-band dimensions include: anxious/depressed, withdrawn/depressed, somatic complaints, social problems, thought problems, attention problems, rule-breaking behavior, and aggressive behavior. Three broad-band dimensions include: internalizing behaviors, externalizing behaviors, and total problem behaviors. Internal consistency ranges from .56 to .92 and one-week test-retest reliability correlations range from .63 to .97 (Achenbach, 1990). Elevated scores indicate increased total problem behaviors and borderline clinical ( $T$-scores of 65-69) and clinical ( $T$-scores of 70 or more) ranges for each subscale. For the present study, internalizing, externalizing, and total behavior problems scores were utilized during analyses.

The Revised Conflict Tactics Scale (CTS2; Straus et al., 1996). (Appendix D). The CTS2 is a 78-item measure designed to assess the amount and severity of violence in a relationship. Ten subscales include: female and male negotiation, female and male sexual violence, female and male psychological violence, female and male injury, and female and male physical violence. Internal consistency ranges from .79 to .95 and the CTS2 is a widely used and validated instrument (Straus et al., 1996). Caregivers reported the frequency of whether they or their partners have demonstrated certain violent behaviors within the previous year. For the present study, female and male psychological violence, and female and male physical violence scores were utilized during analyses.

Brief Symptom Inventory (BSI; Derogatis, 1993). (Appendix E). The BSI is a 53-item measure designed to assess psychological symptoms over the previous seven-days. Nine narrowband dimensions include: somatization, obsessive-compulsive, interpersonal sensitivity, depression, anxiety, hostility, phobic anxiety, paranoid ideation, and psychoticism. Three broad- 
band dimensions include: general severity, positive symptoms, and global severity index. The BSI has internal reliability that ranges from .71 to .85 and test-retest reliability that ranges from .68 to .91 (Derogatis \& Melisaratos, 1983). Elevated scores indicate increased problem behaviors and borderline clinical ( $T$-scores of 60-62) and clinical ( $T$-scores in excess of 62$)$ ranges for each scale. For the present study, the global severity index score was utilized during analysis.

Child Abuse Potential Inventory-IV (CAP; Milner, 1986). (Appendix F). The CAP is a 160-item measure designed to screen for the potential to commit child physical abuse. Six narrow-band dimensions include: distress, rigidity, unhappiness, problems with child and self, problems with family, and problems with others. One broad-band dimension is physical child abuse. Internal consistency ranges from .92 to .96 for controls and .95 to .98 for abusers (Milner, 1986). Test-retest reliability correlations range from .91 (one-day interval) to .75 (three-month interval) (Milner, 1986). Elevated scores indicate increased levels of potential to commit child physical abuse. For the present study, the physical child abuse score was utilized during analysis. Eyberg Child Behavior Inventory (ECBI; Eyberg \& Pincus, 1999). (Appendix G). The ECBI is a 36-item measure designed to assess the frequency and severity of disruptive behaviors of children 2 to16-years-of-age in the home environment. Two broad-band dimensions include intensity and problem. Internal consistency ranges from .88 to .95 and test-retest reliability range from .86 to .88 (Eyberg, 1999). Elevated scores indicate increased levels of child behavior problems and clinical ( $T$-scores above 60$)$ ranges for each scale. For the present study, the intensity and problems scores were utilized during analyses.

Dyadic Parent-Child Interaction Coding System-III (DPICS-III; Eyberg et al., 2004). (Appendix H). The DPICS-III is a behaviorally orientated coding system that may be utilized by clinicians to assess the quality of parent-child interactions. When utilized during an intake 
assessment, the DPICS-III consists of three structured components: child-directed activity, parent-directed activity, and clean-up activity. When utilized during weekly PCIT sessions, DPICS-III provides information regarding parents' and child's particular strengths and weaknesses and provides data to guide each treatment coaching session. DPICS-III has demonstrated both reliability and validity (Eyberg et al., 2004).

Prior to coding live PCIT treatment sessions, research assistants were trained according to the standards set forth in the DPICS-III Workbook and obtained a score of $80 \%$ or above on quizzes regarding the behaviors assessed during treatment. Additionally, research assistants were required to achieve inter-rater reliability kappas of .70 or higher for each target behavior (parent Positive Talk, parent Negative Talk, and Child Compliance) with a PCIT Master Trainer. It was determined that if kappa's on live observations fell below .70 at any point during the duration of the research project, therapists would retrain with a PCIT Master Trainer until kappa's exceeded .70. Interrater agreement occurred for $45 \%$ of coding sessions.

Three scores were derived from data gathered from DPICS-III and used during analyses: parent Positive Talk, parent Negative Talk, and Child Compliance. Positive Talk consists of positive parental verbal behaviors such as labeled praise, behavioral descriptions, and reflections of child language. Negative Talk consists of negative parental verbal behaviors such as criticisms and sarcasm directed toward the child. Child Compliance consists of child physical compliance to parental requests when provided with an opportunity to follow-through with their requests. For the present study, Positive Talk, Negative Talk and Child Compliance were utilized during analyses.

Parenting Stress Index - Short Form (PSI-SF; Abidin, 1995). (Appendix I). The PSI-SF consists of 36-items designed to identify stress in parents of children from 1-month to 12-years- 
of-age. Three narrow-band dimensions include: parental distress, parent-child dysfunctional interaction, and difficult child. One broad-band dimension include is total stress. Elevated scores indicate increased parenting stress and borderline clinical ( $T$-scores of 81-84) and clinical ( $T$ scores in excess of 85) ranges for each scale. For the present study, the total stress score was utilized during analyses.

Therapy Attitude Inventory (TAI; Eyberg, 1993; Eyberg \& Johnson, 1974). (Appendix J). The TAI consists of 10-items designed to assess parental satisfaction with family therapy. One narrow band dimension is the total score. Elevated scores indicate increased levels of satisfaction regarding treatment and improvement of previously identified child behavior problems. External validity ranges from .36 to .49 and test-retest reliabilities are reported at .85 (Brestan, Jacobs, Rayfield, \& Eyberg, 1999). For the present study, the total score was utilized during analyses.

Results

\section{Preliminary Analyses}

In total, data collected for 49 children as reported by 49 caregivers were included in data analysis. To combat missing data, study personnel reviewed assessments prior to compensating participants. If missing data existed, participants were asked if the item was intentionally left blank. Missing data were minimal with the majority of participants omitting no items. If a caregiver omitted more than $10 \%$ of responses on any given measure, data for that measure alone was not considered in further analyses; no participants met this criterion. However, three female participants did refuse to complete The Revised Conflict Tactics Scale reporting verbally that they felt uncomfortable reporting on their prior experiences with IPV. Missing data points for the Child Behavior Checklist, The Revised Conflict Tactics Scale, Brief Symptom Inventory, Child Abuse Potential Inventory, and Eyberg Child Behavior Inventory were replaced with 
overall means for individual items on that measure (Acock, 2005; Roth, 1994). Missing data points for the Dyadic Parent-Child Interaction Coding System-III were not replaced with overall means for individual items on that measure. Data were initially screened for the presence of outliers and problems of skew or kurtosis. These explorations indicated that two variables, posttreatment male-to-female physical and pre-treatment female-to-male physical, each had 1 score that resulted in outliers, skew, or kurtosis which may have affected the distribution of participants' responses. After reviewing the data, these scores were included in the original dataset as they were consistent with the participants' report of other forms of IPV and accurately reflected participants' experiences with IPV.

A power analysis (G*Power 3; Faul, Erdfelder, Lang, \& Buchner, 2007), utilizing methods advocated by Cohen (1988) was conducted and, using a power criterion of .80, it was determined that a sample of 42 caregiver-child dyads would be adequate to detect a large effect size in this research project. According to Cohen (1992), .20 is a small effect size, .50 is a medium effect size, and .80 is a large effect size. Previous research examining the effect sizes of PCIT have demonstrated large effect sizes with reference to child behavior $(d=0.61-1.45)$, classroom report $(d=1.21-1.57)$, and parent behavior and functioning $(d=0.76-5.67)$ (Thomas \& Zimmer-Gembeck, 2007). As a result, the current sample size is considered to have adequate power for detecting large effect sizes.

\section{Descriptive Statistics}

Demographic characteristics of this sample are presented in Table 1. The analyses presented in this document are based upon a sample of 49 caregivers ( 41 females and 8 males) who participated in this research project. Participants were recruited from The Family Junction, Inc; a community outreach organization located in Cumberland, Maryland which was previously 
discussed in this document. The majority of caregivers in this study were Caucasian (93.9\% for females, $77.6 \%$ for males), followed by African-American (2.0\% for females, $14.3 \%$ for males), and multiple ethnicities (4.1\% for males). Overall, caregivers in this study ranged from 17.5 to 54.0 years of age $(M=30.29, S D=7.45)$. Female caregivers ranged from 17.4 to 52.5 years of age $(M=29.3, S D=6.36)$, while male caregivers ranged from 22.9 to 54.0 years of age $(M=$ $35.4, S D=10.68)$. The majority of participants reported that they were either cohabitating with their partner $(28.6 \%)$ or married $(28.6 \%)$, followed by separated $(14.3 \%)$, divorced $(12.2 \%)$ or single (12.2\%), and remarried following divorce (4.1\%).

Regarding educational attainment, participants reported having completed junior high school (4.9\% women, $12.5 \%$ males), partial high school (26.8\% female, $25.0 \%$ male), high school or obtaining a general education diploma (34.1\% female, $37.5 \%$ male), specialized training or partially fulfilling college requirements (29.3\% female, $12.5 \%$ male), college graduate $(2.4 \%$ female, $12.5 \%$ male), and professional degree (2.4\% female, $12.5 \%$ male). Regarding socioeconomic status, participants reported being in Category 1 (49.0\%), Category 2 (36.7\%), Category $3(2.0 \%)$, Category $4(6.1 \%)$, and Category $5(2.0 \%)$. Caregivers in this study did not demonstrate elevated levels of psychopathology as indicated by the BSI. Assessment measures were completed for 49 children (19 female and 30 male). The majority of children were Caucasian (81.6\%), followed by multiple-ethnicities (18.4\%). Children in this study ranged from 1.3 to 15.25 years of age $(M=6.78, S D=3.35)$. Two children fell outside of the predetermined age range for this study. However, they were included in analyses as the target of behavior change in this study was actually the caregiver and not the child. Overall means and standard deviations for assessment measures are presented in Table 2. 
Major Analyses

Hypothesis 1. Child Abuse and Neglect. Potential child abuse and neglect risk as indicated by the Child Abuse Potential Inventory (CAP; Milner, 1986) will be related to treatment condition. It was hypothesized that the CAP would decrease more in the Parent-Child Interaction Therapy (PCIT) condition compared to the treatment as usual (TAU) condition from pre- to post-treatment.

To begin, the first analysis that occurred was to determine whether there were significant differences between the PCIT and TAU conditions at pre-treatment on the dependent variable. Analyses indicated no significant differences in CAP physical child abuse scores between treatment conditions at pre-treatment, $F(1,45)=1.91, p=0.17$. Subsequently, a 2 (treatment condition) X 2 (time) repeated measures ANCOVA was utilized to determine if treatment condition was related to CAP physical child abuse scores. Descriptive statistics are shown in Table 3. Analyses indicated that CAP physical child abuse scores did have a greater decrease from pre- to post-treatment in the PCIT condition compared to the TAU condition, $F(2,41)=$ 12.12, $p=0.00$. Specifically, results indicated that treatment condition was related to greater decreases in CAP child physical abuse scores from pre- to post-treatment.

Hypothesis 2. Intimate Partner Violence. Intimate partner violence as indicated by the Revised Conflict Tactics Scale (CTS2; Straus, Hamby, Boney-McCoy, \& Sugarman, 1996) will be related to treatment condition. It was hypothesized that the CTS2 would decrease more in the PCIT condition compared to the TAU condition from pre-to post-treatment.

A series of 2 (treatment condition) X 2 (time) repeated measures ANCOVA's were utilized to determine if treatment condition was related to CTS2 male-to-female psychological 
violence scores, female-to-male psychological violence scores, male-to-female physical violence scores, and female-to-male physical violence scores.

Hypothesis 2a. Intimate Partner Violence. To begin, the first analysis that occurred was to determine whether there were significant differences between the PCIT and TAU conditions at pre-treatment on the dependent variable. Analyses indicated no significant differences in CTS2 male-to-female psychological violence scores between treatment conditions at pre-treatment, $F$ $(1,41)=0.09, p=0.76$. Subsequently, a 2 (treatment condition) $\mathrm{X} 2$ (time) repeated measures ANCOVA was utilized to determine if treatment condition was related to CTS2 male-to-female psychological violence scores. Descriptive statistics are shown in Table 4. Analyses indicated that CTS2 male-to-female psychological violence scores did not have a greater decrease from pre- to post-treatment in the PCIT condition compared to the TAU conduction, $F(2,39)=0.68$, $p=0.51$. Specifically, results indicated that treatment condition was not related to greater decreases in CTS2 male-to-female psychological violence scores from pre- to post-treatment.

Hypothesis $2 b$. Intimate Partner Violence. To begin, the first analysis that occurred was to determine whether there were significant differences between the PCIT and TAU conditions at pre-treatment on the dependent variable. Analyses indicated no significant differences in CTS2 female-to-male psychological violence scores between treatment conditions at pre-treatment, $F$ $(1,41)=0.01, p=0.91$. Subsequently, a 2 (treatment condition) X 2 (time) repeated measures ANCOVA was utilized to determine if treatment condition was related to CTS2 female-to-male psychological violence scores. Descriptive statistics are shown in Table 5. Analyses indicated that CTS2 female-to-male psychological violence scores did not have a greater decrease from pre- to post-treatment in the PCIT condition compared to the TAU conduction, $F(2,39)=1.78$, 
$p=0.18$. Specifically, results indicated that treatment condition was not related to greater decreases in CTS2 female-to-male psychological violence scores from pre- to post-treatment.

Hypothesis 2c. Intimate Partner Violence. To begin, the first analysis that occurred was to determine whether there were significant differences between the PCIT and TAU conditions at pre-treatment on the dependent variable. Analyses indicated no significant differences in CTS2 male-to-female physical violence scores between treatment conditions at pre-treatment, $F(1,41)$ $=0.84, p=0.36$. Subsequently, a 2 (treatment condition) $\mathrm{X} 2$ (time) repeated measures ANCOVA was utilized to determine if treatment condition was related to CTS2 male-to-female physical violence scores. Descriptive statistics are shown in Table 6. Analyses indicated that CTS2 male-to-female physical violence scores did not have a greater decrease from pre- to posttreatment in the PCIT condition compared to the TAU conduction, $F(2,39)=0.49, p=0.61$. Specifically, results indicated that treatment condition was not related to greater decreases in CTS2 male-to-female physical violence scores from pre- to post-treatment.

Hypothesis $2 d$. Intimate Partner Violence. To begin, the first analysis that occurred was to determine whether there were significant differences between the PCIT and TAU conditions at pre-treatment on the dependent variable. Analyses indicated no significant differences in CTS2 female-to-male physical violence scores between treatment conditions at pre-treatment, $F(1,41)$ $=1.07, p=0.31$. Subsequently, a 2 (treatment condition) $\mathrm{X} 2$ (time) repeated measures ANCOVA was utilized to determine if treatment condition was related to CTS2 female-to-male physical violence scores. Descriptive statistics are shown in Table 7. Analyses indicated that CTS2 female-to-male physical violence scores did not have a greater decrease from pre- to posttreatment in the PCIT condition compared to the TAU conduction, $F(2,39)=0.93, p=0.40$. 
Specifically, results indicated that treatment condition was not related to greater decreases in CTS2 female-to-male physical violence scores from pre- to post-treatment.

Hypothesis 3. Parental Stress. Parental stress as indicated by the Parenting Stress IndexShort Form (PSI-SF; Abidin, 1995) will be related to treatment condition. It was hypothesized that the PSI-SF total stress score would decrease more in the PCIT condition compared to the TAU condition from pre- to post-treatment.

To begin, the first analysis that occurred was to determine whether there were significant differences between the PCIT and TAU conditions at pre-treatment on the dependent variable. Analyses indicated no significant differences in PSI-SF total stress score between treatment conditions at pre-treatment, $F(1,45)=2.23, p=0.14$. Subsequently, a 2 (treatment condition) $\mathrm{X}$ 2 (time) repeated measures ANCOVA was utilized to determine if treatment condition was related to PSI-SF total stress scores. Descriptive statistics are shown in Table 8. Analyses indicated that PSI-SF total stress scores did have a greater decrease from pre- to post-treatment in the PCIT condition compared to the TAU conduction, $F(2,42)=3.09, p=0.05$.

Hypothesis 4. Child Behavior. Child behavior as indicated by the Child Behavior Checklist (CBCL; Achenbach, 2001; Achenbach \& Rescola, 2000) and Dyadic Parent-Child Interaction Coding System-III (DPICS-III; Eyberg, McDiarmid Nelson, Duke, \& Boggs, 2004) Child Compliance category will be related to treatment condition. It was hypothesized that the CBCL would decrease more in the PCIT condition compared to the TAU condition from pre-to post-treatment and that Child Compliance would be greater in the PCIT condition than in the TAU condition at post-treatment.

A series of 2 (treatment condition) X 2 (time) repeated measures ANCOVA's were utilized to determine if treatment condition was related to Child Behavior Checklist internalizing, 
externalizing, and total behaviors scores. In addition, a paired samples t-test was conducted to determine whether Dyadic Parent-Child Interaction Coding System-III Child Compliance scores were greater in the PCIT condition than in the TAU condition at post-treatment.

Hypothesis 4a. Child Behavior. It was hypothesized that the CBCL internalizing behavior problem score would decrease more in the PCIT condition compared to the TAU condition from pre- to post-treatment.

To begin, the first analysis that occurred was to determine whether there were significant differences between the PCIT and TAU conditions at pre-treatment on the dependent variable. Analyses indicated significant differences in CBCL internalizing behavior problem scores between treatment conditions at pre-treatment, $F(1,41)=6.01, p=0.02$. As a significant pretreatment difference was present, an ANCOVA was conducted to control for the associated variance. Subsequently, a 2 (treatment condition) X 2 (time) repeated measures ANCOVA was utilized to determine if treatment condition was related to changes in CBCL internalizing behavior problem scores across time controlling for the influence of CBCL internalizing scores at pre-treatment upon post-treatment assessment. Descriptive statistics are shown in Table 9. The corrected model indicated a significant difference in CBCL internalizing behavior scores between PCIT and TAU treatment conditions at post-treatment, $F=(2,38) 9.01, p=.001$. These results indicate that treatment condition was related to CBCL internalizing behavior scores. Specifically, participants in the PCIT treatment condition reported greater decreases in CBCL internalizing behavior scores from pre- to post-treatment than participants in the TAU condition. Hypothesis 4b. Child Behavior. It was hypothesized that the CBCL externalizing behavior problem scores would decrease more in the PCIT condition compared to the TAU condition from pre- to post-treatment. 
To begin, the first analysis that occurred was to determine whether there were significant differences between the PCIT and TAU conditions at pre-treatment on the dependent variable. Analyses indicated no significant differences in CBCL externalizing behavior problem scores between treatment conditions at pre-treatment, $F(1,41)=2.92, p=0.10$. Subsequently, a 2 (treatment condition) X 2 (time) repeated measures ANCOVA was utilized to determine if treatment condition was related to CBCL externalizing behavior problem score. Descriptive statistics are shown in Table 10. Analyses indicated that CBCL externalizing behavior problem scores did have a greater decrease from pre- to post-treatment in the PCIT condition compared to the TAU conduction, $F(2,38)=18.96, p=0.00$. These results indicate that treatment condition was related to CBCL externalizing behavior scores. Specifically, participants in the PCIT condition reported greater decreases in CBCL externalizing behavior scores from pre- to posttreatment than participants in the TAU condition.

Hypothesis 4c. Child Behavior. It was hypothesized that the CBCL total behavior problems would decrease more in the PCIT condition compared to the TAU condition from preto post-treatment.

To begin, the first analysis that occurred was to determine whether there were significant differences between the PCIT and TAU conditions at pre-treatment on the dependent variable. Analyses indicated no significant differences in CBCL total behavior problems scores between treatment conditions at pre-treatment, $F(1,41)=2.30, p=0.14$. Subsequently, a 2 (treatment condition) X 2 (time) repeated measures ANCOVA was utilized to determine if treatment condition was related to CBCL total behavior problems scores. Descriptive statistics are shown in Table 11. Analyses indicated that CBCL total behavior problems scores did have a greater decrease from pre- to post-treatment in the PCIT condition compared to the TAU conduction, $F$ 
$(2,38)=15.88, p=0.00$. These results indicate that treatment condition was related to CBCL total behavior scores. Specifically, participants in the PCIT condition reported greater decreases in CBCL total behavior scores from pre- to post-treatment than participants in the TAU condition.

Hypothesis 4d. Child Behavior. It was hypothesized that the DPICS-III Child Compliance score would decrease in the PCIT condition from pre- to post-treatment.

At post-treatment assessment, DPICS-III Child Compliance scores were greater in the PCIT condition $(M=0.90, S D=.25)$ compared to the TAU condition $(M=0.68, S D=.28)$. Descriptive statistics are shown in Table 12. Though investigatory, these results indicate that treatment condition may be related to DPICS-III Child Compliance scores. Specifically, children in the PCIT treatment condition display greater rates of DPICS-III Child Compliance scores at post-treatment than children in the TAU condition.

Hypothesis 4e. It was hypothesized that ECBI Intensity score would decrease more in the PCIT condition compared to the TAU condition from pre-to post-treatment.

To begin, the first analysis that occurred was to determine whether there were significant differences between the PCIT and TAU conditions at pre-treatment on the dependent variable. Analyses indicated no significant differences in ECBI Intensity score between treatment conditions at pre-treatment, $F(1,43)=3.50, p=0.07$. Subsequently, a 2 (treatment condition) $\mathrm{X}$ 2 (time) repeated measures ANCOVA was utilized to determine if treatment condition was related to ECBI Intensity scores. Descriptive statistics are shown in Table 13. Analyses indicated that ECBI Intensity scores did have a greater decrease from pre- to post-treatment in the PCIT condition compared to the TAU conduction, $F(2,41)=24.16, p=0.00$. These results indicate that treatment condition was related to ECBI Intensity scores. Specifically, participants 
in the PCIT condition reported greater decreases in ECBI Intensity scores from pre- to posttreatment than participants in the TAU condition.

Hypothesis 4f. It was hypothesized that ECBI Problem score would decreased more in the PCIT condition compared to the TAU condition from pre- to post-treatment.

To begin, the first analysis that occurred was to determine whether there were significant differences between the PCIT and TAU conditions at pre-treatment on the dependent variable. Analyses indicated no significant differences in ECBI Problem scores between treatment conditions at pre-treatment, $F(1,42)=2.22, p=0.14$. Subsequently, a 2 (treatment condition) $\mathrm{X}$ 2 (time) repeated measures ANCOVA was utilized to determine if treatment condition was related to ECBI Severity scores. Descriptive statistics are shown in Table 14. Analyses indicated that ECBI Severity scores did have a greater decrease from pre- to post-treatment in the PCIT condition compared to the TAU conduction, $F(2,40)=18.29, p=0.00$. These results indicate that treatment condition was related to ECBI Problem scores. Specifically, participants in the PCIT condition reported greater decreases in ECBI Problem scores from pre- to post-treatment than participants in the TAU condition.

Hypothesis 5. Parenting Behavior. Parenting behavior as indicated by the DPICS-III Positive Talk and Negative Talk categories will be related to treatment condition.

Descriptions and paired samples t-tests were utilized to determine if Dyadic Parent-Child Interaction Coding System-III Positive Talk and Negative Talk scores decreased from pre- to post-treatment in the PCIT condition.

Hypothesis 5a. Parenting Behavior. It was hypothesized that DPICS-III Positive Talk scores would increase in the PCIT condition from pre-to post-treatment. 
Only data from the PCIT condition was utilized during analyses due to a small sample size in the TAU condition. While child participation was required for the PCIT condition, this was not necessarily required for the TAU condition. As a result, the sample size in the TAU condition did not meet the assumptions of the statistical analyses (Repeated Measures ANCOVA) and was not able to be analyzed. A paired samples t-tests was utilized to determine if DPICS-III Positive Talk scores would decrease from pre- to post-treatment in the PCIT condition. Descriptive statistics are shown in Table 15. Analyses indicated that DPICS-III Positive Talk scores in the PCIT condition did have a significant increase from pre- $(M=11.71$, $S D=7.33)$ to post-treatment $(M=18.89, S D=12.46), t(18)=-2.90, p=.01$. Specifically, results indicated that DPICS-III Positive Talk scores did significantly increase in the PCIT condition from pre- to post-treatment.

Hypothesis 5b. Parenting Behavior. It was hypothesized that DPICS-III Negative Talk score would decrease in the PCIT condition from pre- to post-treatment.

Only data from the PCIT condition was utilized during analyses due to a small sample size in the TAU condition. While child participation was required for the PCIT condition, this was not necessarily required for the TAU condition. As a result, the sample size in the TAU condition did not meet the assumptions of the statistical analyses (Repeated Measures ANOVA) and was not able to be analyzed. A t-test was utilized to determine if DPICS-III Negative Talk scores would decrease from pre- to post-treatment in the PCIT condition. Descriptive statistics are shown in Table 16. Analyses indicated that DPICS-III Negative Talk scores in the PCIT condition did have a significant decrease from pre- $(M=6.03, S D=4.19)$ to post-treatment $(M=$ $2.44, S D=2.03), t(19)=6.43, p=.00$. Specifically, results indicated that DPICS-III Negative Talk scores did decrease significantly in the PCIT condition from pre- to post-treatment. 
While results did not indicate a significantly greater decrease in CAN between PCIT and TAU, analyses did indicate a decrease in CAN in the PCIT condition which may be accounted for by the combination of caregiver-child in treatment, caregiver level of involvement in treatment, content of treatment material, and treatment group construction.

First, the combination of caregiver-child involvement in the PCIT group may be responsible for decreases in CAN. The TAU group consisted of two separate groups, one for children who participated in non-therapeutic play activities with child care staff, and one for caregivers who attended separate lectures on a variety of topics. Alternatively, PCIT groups consisted of conjoint parent-child treatment sessions designed to improve individual parent-child relationships and increase cooperation and cohesion of all family members. While caregiver retraining is a central component of many treatment programs for caregivers who have been abusive towards their children in order to decrease recidivism (Chaffin et al., 2004; Cohen et al., 2006), treatments should incorporate both caregiver and child(ren) in the therapeutic process (Borrego et al., 1999; Chaffin et al., 2004; Timmer et al., 2005b) in order to maximize effectiveness. Caregivers-child(ren) in the PCIT condition learned how to work together as a team, enjoyed developing a positive relationship, and had a mutual goal (i.e., skill mastery) to achieve. The inclusion of caregivers and child(ren) may impact the parent-child dynamic more when combined versus when treated as separate entities.

Second, caregiver level of involvement and participation in the PCIT program may be responsible for decreases in CAN. The TAU condition was conducted primarily as a lecture format with minimal interaction required from participants. Caregivers in the PCIT condition were not merely passive consumers of a program aimed at reducing CAN recidivism. Instead, involvement in the PCIT condition necessitated caregivers' active involvement in every 
component of the therapeutic process. Caregivers were required to participate in the didactic sessions, including asking and answering questions related to treatment and management of child behaviors. They also (a) were coded and coached for successful implementation of CDI and PDI skill mastery with their child(ren) at each session, (b) were required to complete daily, 5-minute, PCIT sessions at home with their child(ren) and return homework sheets on a weekly basis, and (c) were required to receive detailed feedback regarding their strengths and areas in need of improvement on a weekly basis. This intensive level of teaching and caregiver involvement may have facilitated a deep, long-lasting understanding of the theory supporting the applied components of PCIT. Understanding the rationale for these components, rather than merely accepting the components at face value, may have assisted in the acquisition of these skills during the treatment process.

Third, the content of the material included in the PCIT condition may be responsible for decreases in CAN. The TAU condition did not incorporate any specific skills that research has demonstrated are effective in reducing CAN. Alternatively, the PCIT condition taught several skill-sets instrumental in the reduction of CAN (Chaffin et al., 2004). Specifically, caregivers were taught to identify and verbalize their child's positive verbal and physical behaviors in a reinforcing manner (Timmer et al., 2005a; Timmer et al., 2005b; Timmer et al., 2006). During this phase, children were taught what behaviors were and were not appropriate through differential reinforcement (Timmer et al., 2005a; Timmer et al., 2005b; Timmer et al., 2006). Next, caregivers were taught to identify and reduce their own negative verbal and physical behaviors that may exacerbate their child's negative behaviors. Furthermore, caregivers were taught, modeled, and coached on how to implement non-violent discipline strategies to reduce inappropriate child behaviors coupled with strategies to manage their own negative emotional 
and physical reactions to their child's behaviors Chaffin et al., 2004; Timmer et al., 2005a; Timmer et al., 2005b; Timmer et al., 2006).

In summary, both caregiver and child were aware of all aspects of the newly developed strategy eliminating caregiver and child confusion and decreasing caregiver and child stress. The multitude of skills caregivers developed during treatment naturally built upon each other and increased in quality and quantity during the course of treatment due to repeated exposure and extensive coaching in skill acquisition. Alternatively, the TAU condition may have briefly discussed these concepts, but did not teach or model them in-depth.

Lastly, the structure of the PCIT condition groups may be responsible for decreases in CAN. TAU groups were not formed anew. Instead, new caregivers were simply incorporated into classes that were continuously conducted. For example, some caregivers would begin at session 1 , some at session 6 , and some at session 12 . There was no beginning or end point for the group or a time-frame regarding class completion. Conversely, caregivers enrolled in the PCIT condition began and ended treatment with an entire class of other caregivers. Caregivers began with the same level of knowledge at pre-treatment and developed group cohesion where they were able to support each other during the treatment process. This support could be observed when caregivers coached other caregivers in implementing the CDI skills, implementing a PDI time-out procedure, or congratulating each other on skills mastery. Caregivers in the PCIT condition had mutual goals (i.e., skill mastery), provided empathy and support for each other, and coordinated transportation and childcare during PCIT groups (Niec et al., 2005). 


\section{Exploratory Analyses}

\section{Child Gender and Age}

Independent samples t-tests did not reveal any significant differences between female and male children on any of the dependent variables (child abuse and neglect, intimate partner violence, parental stress, child behavior, and parenting behavior) in this study (all $p$ 's $>.05$ ). Pearson's correlation coefficients did not reveal any significant relationships regarding child age and any dependent variables (child abuse and neglect, intimate partner violence, parental stress, child behavior, and parenting behavior) in this study (all p's > .05).

\section{Child Ethnicity}

Independent samples t-tests did reveal significant differences between Caucasian and non-Caucasian children for two of the dependent variables (child abuse and neglect, intimate partner violence, parental stress, child behavior, and parenting behavior) in this study. Results indicated a significant difference between Caucasian children $(M=2.12, S D=5.03)$ and nonCaucasian children $(M=9.57, S D=12.59)$ for post-treatment CTS2 male-to-female physical violence, $t(39)=-2.65, p=.01$. These results indicate that Caucasian children were exposed to less male-to-female physical violence than their non-Caucasian counterparts at post-treatment. Results also indicated a significant relationship between Caucasian children $(M=50.43, S D=$ 12.14) and non-Caucasian children $(M=60.43, S D=10.77)$ for post-treatment CBCL internalizing behavior problems scores, $t(40)=-2.02, p=.05)$. This indicates that Caucasian children were less likely to experience internalizing behavior problems at post-treatment than non-Caucasian children. Independent samples t-tests did not reveal any other significant differences between Caucasian and non-Caucasian children and any other dependent variables (child abuse and neglect, parental stress, and parenting behavior) in this study (all p's >.05). 
Socioeconomic Status (SES)

Pearson's correlation coefficients were utilized to determine if socioeconomic status was related to the dependent variables (child abuse and neglect, intimate partner violence, parental stress, child behavior, and parenting behavior) in this study. Bivariate correlational analysis indicated a significant correlation between socioeconomic status and post-treatment DPICS-III Negative Talk scores, $r(19)=.56, p=.012$, denoting that increases in socioeconomic status were related to increases in negative parental communication scores at post-treatment. Pearson's correlation coefficients did not reveal any other significant relationships between socioeconomic status and any other dependent variables (child abuse and neglect, intimate partner violence, parental stress, and child behavior) in this study (all $p$ 's $>.05$ ).

\section{Maternal Education}

Pearson's correlation coefficients were utilized to determine if maternal education was related to the dependent variables (child abuse and neglect, intimate partner violence, parental stress, child behavior, and parenting behavior) in this study. Bivariate correlational analyses indicated a significant correlation between maternal education and post-treatment PSI-SF total stress scores, $r(46)=.29, p=.05$, denoting that increases in maternal education were related to increases in parental stress scores at post-treatment. Pearson's correlation coefficients did not reveal any other significant relationships between maternal education and any other dependent variables (child abuse and neglect, intimate partner violence, child behavior, and parenting behavior) in this study (all p's $>.05$ ).

\section{Paternal Education}

Pearson's correlation coefficients were utilized to determine if paternal education was related to the dependent variables (child abuse and neglect, intimate partner violence, parental 
stress, child behavior, and parenting behavior) in this study. Pearson's correlation coefficients did not reveal any other significant relationships between paternal education and any dependent variables (child abuse and neglect, intimate partner violence, parental stress, child behavior, and parenting behavior) in this study (all p's $>.05$ ).

Caregiver Age

Pearson's correlation coefficients were utilized to determine if caregiver age was related to the dependent variables (child abuse and neglect, intimate partner violence, parental stress, child behavior, and parenting behavior) in this study. Bivariate correlational analyses indicated a significant correlation between caregiver age and pre-treatment CBCL internalizing behavior problem scores, $r(43)=-.36, p=.02$, denoting that decreases in caregiver age were related to increases in child internalizing behaviors at pre-treatment. Pearson's correlation coefficients did not reveal any other significant relationships between caregiver age and any other dependent variables (child abuse and neglect, intimate partner violence, parental stress, and parenting behavior) in this study (all $p$ 's $>.05$ ).

\section{Therapy Attitude Inventory}

Independent samples t-tests did not reveal significant differences between the PCIT condition and TAU condition regarding satisfaction with therapy programs in this study. Results indicated that the PCIT condition $(M=44.05, S D=5.64)$ and TAU condition $(M=42.17, S D=$ 7.69) were not significantly different at post-treatment, $t(41)=0.89, p=0.38$. These results indicate no differences in satisfaction with therapy programs between treatment conditions.

\section{Discussion}

This study aimed to replicate previous findings that have demonstrated that PCIT is an effective treatment at reducing CAN. This study also sought to extend those findings by 
exploring the impact of PCIT upon IPV. Furthermore, the present investigation attempted to advance knowledge by evaluating PCIT in a community setting, using traditional resources that exist in community agencies. As a result, PCIT was provided without the one-way mirror, hearing-aid device, or specially designed time-out room that are typically integral to PCIT's implementation. Additionally, consistent with the mandate of community mental health to service large numbers of families in a cost effective manner, PCIT was conducted in this study using a group format. When evaluating the study overall, the results can best be described as mixed. There were many positive findings suggesting that PCIT can be provided successfully in a community setting without special equipment, and that group PCIT can be effective across many domains (e.g., child behavior, parental skill acquisition, parenting stress). Yet, the study did not provide clear support for PCIT as a treatment for decreasing IPV, and the effectiveness of PCIT for decreasing child abuse potential remains unclear. The discussion below examines each of the study's findings and possible implications, while delineating the methodological limitations that must be considered when interpreting the results.

\section{Child Abuse and Neglect}

There was a significantly greater decrease in child abuse potential scores on the Child Abuse Potential Inventory from pre- to post-treatment for the PCIT condition as compared to the TAU condition. These results were anticipated as previous research has demonstrated that families with a history of CAN who receive PCIT treatment services report decreases in potential child abuse and neglect from pre- to post-treatment (Borrego et al., 1999; Timmer et al., 2005a; Timmer et al., 2005b; Timmer et al., 2006). Results also indicated that PCIT did produce greater changes in parenting stress and parenting behaviors that may result in decreased CAN. Most notably, findings revealed a greater decrease in parenting stress, increase in positive talk, and 
decrease in negative talk in the PCIT condition compared to the TAU condition. In addition, findings revealed a greater decrease in severity and intensity of child behaviors problems in the PCIT condition compared to the TAU condition. These aspects of parenting and child behavior problems may be components that impact CAN. For example, parents who experience less stress and speak more positively and less negatively with their children may be less likely to partake in CAN activities. In fact, Chaffin et al. (2004) found that parental negative verbalizations were a mediator for future recidivism of $\mathrm{CAN}$, such that greater reductions in negative comments by parents in PCIT were related to fewer future child abuse and neglect reports. With respect to child behavior, children who have fewer severe and intense behaviors problems are more likely to have a positive relationship with their caregivers and decreased likelihood of engaging in problem behaviors that may result in CAN (Urquiza \& McNeil, 1996). Taken together, these findings suggest PCIT was effective at reducing child abuse and neglect potential in this study.

\section{Intimate Partner Violence}

Contrary to the hypothesis, the PCIT group did not have a significantly greater decrease in IPV scores than the TAU group from pre- to post-treatment on any of the following four measures: male-to-female psychological violence, female-to-male psychological violence, maleto-female physical violence, or female-to-male physical violence. However, while not significant, it is interesting to note that all four of these measures changed for both the PCIT and TAU conditions in the anticipated directions. In the PCIT condition, the means for all four of these scales of IPV decreased from pre- to post-treatment. In contrast, the means for the TAU condition on all four of these IPV scales increased from pre- to post-treatment.

This study was a preliminary investigation of the effects of PCIT and TAU in reducing IPV in families with a history of co-morbid IPV and CAN. While none of the hypotheses were 
supported, results of the CTS2 female and male psychological violence and female and male physical violence scores were consistently in the anticipated direction for both groups. Of note, IPV was not specifically addressed during either PCIT or TAU treatment condition, but was merely assessed at pre- and post-treatment. There are several possible explanations for the lack of significant findings, such as questionable generalization of PCIT skills, questionable validity of the IPV self reports, lack of sufficient statistical power to detect actual differences, and scale or metric issues that may have influenced analyses.

The first issue with regard to the lack of significant findings in the area of IPV pertains to the questionable strength of skill generalization. Previous research has indicated that skills taught during PCIT treatment may generalize to untreated siblings (Brestan et al., 1997). An extension of this finding would be that these skills may also generalize to other family members, and in this study, the caregiver-caregiver relationship. However, the population examined by Brestan and colleges (1997) did not report any history of CAN nor IPV. There is a high probability that families with a history of CAN or IPV have much different family interaction patterns compared to families without a history of CAN and IPV. As such, PCIT skills may generalization more in families without a history of co-morbid family violence compared to families with a history of co-morbid family violence. In order to facilitate skills generalization with CAN and IPV populations, it may be necessary to modify the PCIT protocol to include components such as psychosocial education regarding IPV and caregiver-caregiver communication training in order for PCIT skills to generalize. Modifications to the PCIT protocol have been successful with children who experience Separation Anxiety Disorder (SAD) and have included such components as Bravery Directed Interaction, lengthening number of treatment sessions, and modifying DPCIS criteria, and the number of pre-treatment coding 
segments (Pincus, Eyberg, \& Choate, 2005). The inclusion of modifications designed to reduce IPV specifically may have resulted in significant findings.

The second issue regarding the lack of findings with IPV pertains to the questionable validity of self report measures of family violence. After all, caregivers were highly motivated to present a positive perception of themselves and their family during the course of treatment. Families in both the PCIT and TAU conditions were court-ordered to attend treatment due to substantiated CAN charges and reunification with their children was dependent upon their successful completion of either program. Hence, reporting IPV may have resulted in an unfavorable report to the court. Therefore, caregivers may have been motivated to under-report or not report violence in their relationships. For example, one caregiver reported no history of violence in her relationship at pre- or post-treatment assessment despite reporting during one session that her partner had been arrested twice the prior night for assaulting her physically and sexually. Also, three female caregivers declined to complete the CTS2 at post-treatment assessment, suggesting that they were not comfortable reporting about their previous IPV experiences. Furthermore, many caregivers reported having multiple consecutive or overlapping romantic relationships during PCIT and TAU treatment. The CTS2 assessed for quantity of IPV experienced during the previous year but did not account for differing levels of violence throughout the year or from multiple partners throughout the year. In addition, definitions of violence and level of acceptability of violence in their interpersonal relationships may differ across participants. Subsequently, all IPV results should be interpreted with caution. Descriptive statistics are shown in Table 17.

The third issue regarding the lack of findings on IPV measures pertains to statistical power due to sample size. Specifically, not all caregivers in either the PCIT or TAU condition 
had ever experienced IPV, some had not been in intimate relationships for the previous year, and others still were involved in concurrent relationships with varying levels of IPV. This greater decreased the same size which in turn impacted statistical power. The original power analysis was based upon finding strong effect sizes. Hence, there may be mild to moderate effects for IPV that were undetected due to lack of statistical power.

Lastly, instructions for the CTS2 indicated to complete the assessment measure based upon IPV experienced during the previous year time-frame. Due to the design of the study, it may have been more appropriate to alter these instructions to assess for the three months prior to pre-treatment and for the three prior to post-treatment, which would have encompassed the time participants were involved in the study. In addition, participants may have also benefited from completing a time-line of major events, such as holidays or personal events, which would have allowed participants to have more concrete anchors regarding frequency and severity of IPV experiences.

\section{Parental Stress}

As hypothesized, results indicated a greater decrease in parental stress scores from pre- to post-treatment in the PCIT condition compared to the TAU condition. These results were anticipated as previous research has demonstrated that families with a history of CAN who receive PCIT treatment services report decreases in parental stress from pre- to post-treatment (Borrego et al., 1999; Timmer et al., 2005a; Timmer et al., 2005b; Timmer et al, 2006).

There are several explanations for significantly greater decreases in parental stress scores in the PCIT condition compared to the TAU condition, such as providing caregivers with psychosocial education, developing support structures, and providing multiple behavior management strategies regarding child behaviors. First, providing caregivers with psychosocial 
education regarding their children's behaviors may have resulted in decreases in parental stress. Children's inappropriate behaviors were addressed, discussed, and normalized during the treatment process. Caregivers were taught developmentally appropriate behavioral expectations. Having more realistic expectations of the children may have altered caregiver perception of the severity and quantity of their children's inappropriate behaviors thereby decreasing their overall stress levels. The TAU condition did not discuss specific ways to manage parental stress regarding caregivers' emotional and physiological reactions to inappropriate child behaviors. In contrast, the PCIT condition explicitly addressed throughout the duration of treatment caregivers' evaluations of inappropriate child behavior, reactions to inappropriate child behavior, evaluation of the effectiveness of the caregivers' reactions in reducing inappropriate child behaviors, developing alternative methods to manage parental reactions, implementing these methods, evaluating them for effectiveness, and making modifications to these behavior management strategies as necessary.

Second, providing caregivers with support from other caregivers may have assisted them in managing their parental stress. As previously stated, the TAU condition consisted of enrolling new caregivers into a pre-existing group. Because caregivers entered at different times, they may not have felt a strong connection with other caregivers in the group and had varying levels of parenting knowledge. In contrast, caregivers in the PCIT condition began and ended treatment together, entered the group with the same level of knowledge, and had a mutual goal. Interaction and discussion of child behavior problems and management strategies were encouraged and required in PCIT sessions. As such, caregivers in the PCIT condition may have been more likely to access other caregivers for support during times of parental stress. 
Lastly, teaching, modeling, and coaching caregivers on the implementation of behavior management strategies for their child's inappropriate behavior may have decreased their parental stress. The TAU condition briefly discussed child behavior management strategies during several treatment sessions. Alternatively, PCIT specifically focused on teaching behavior management strategies such as differential reinforcement of behaviors, re-direction, and time-out. As such, PCIT teaches caregivers multiple strategies that they may implement to manage their child's inappropriate behaviors. Having this toolbox of strategies may allow caregivers to feel more confident that they and their children are prepared to manage inappropriate behaviors, which in turn may, decrease parental stress.

\section{Child Behavior}

As hypothesized, there were significantly greater decreases in the PCIT group's CBCL internalizing, externalizing, and total behavior problem scores as compared to the TAU condition. These results were anticipated as previous research has demonstrated that families with a history of CAN who receive PCIT treatment services report decreases in child internalizing behaviors from pre- to post-treatment (Borrego et al., 1999; Timmer et al., 2005a; Timmer et al., 2005b; Timmer et al., 2006). Of note, mean CBCL internalizing, externalizing, and total behavior problem scores for PCIT and TAU conditions were within the normal behavior range and not the clinically significant range of behavior problems. As hypothesized, there were significantly greater decreases in both ECBI Intensity and ECBI Problem scores in the PCIT condition as compared to the TAU group. These results were anticipated as previous research has demonstrated that families with a history of CAN who receive PCIT treatment services report decreases in ECBI Intensity scores from pre- to post-treatment (Borrego et al., 1999; Timmer et al., 2005a; Timmer et al., 2005b; Timmer et al., 2006). Results on observed 
child compliance are difficult to interpret because of missing data (see discussion on the methodological concerns regarding behavioral observations below). At post-treatment, however, the PCIT group was $90 \%$ compliant during the DPICS observations, a rate that is higher than the normative rate for the DPICS-III (Eyberg et al., 2004), suggesting that the children did not have significant compliance concerns following PCIT.

Taken together, the child behavior results suggest that PCIT was more effective than TAU in reducing children's disruptive behavior. In the TAU condition, treatment was not focused on reducing inappropriate child behavior or increasing appropriate child behavior. Conversely, the PCIT condition did address these specific child behaviors throughout the course of treatment. There are several explanations for the decrease in inappropriate child behavior and the increase in child appropriate behavior such as clear expectations, modeling of respectful behaviors, and perception of child behaviors.

First, children in the TAU condition were not provided with any therapeutic services or psychosocial education regarding behavioral expectations and consequences dependent upon behaviors. On the other hand, children in the PCIT condition were taught clear behavioral expectations and that inappropriate behaviors would result in consistent, immediate consequences (time-out). It follows that these components of PCIT are responsible for children in this condition being less likely to demonstrate inappropriate behaviors resulting in punishment and more likely to demonstrate appropriate behaviors eliciting verbal praise and access to desired privileges from caregivers.

Second, caregivers in the TAU condition were provided with minimal psychosocial education regarding implementing and modeling appropriate behaviors with their children and these strategies were not practiced repeatedly during treatment. On the other hand, caregivers in 
the PCIT condition were taught to model appropriate behaviors such as respectful tone of voice, clear expectations of behaviors, and good manners when providing directives to their children. It reasons that children in the PCIT condition were more likely to comply with directives to cease inappropriate behaviors or to complete a request because when they were treated respectfully and were able to understand their caregivers' expectations.

\section{Parenting Behavior}

As expected, there was a significant increase in DPICS-III Positive Talk scores from preto post-treatment for the PCIT group. These results were anticipated as previous research has demonstrated that families with histories of CAN who receive PCIT treatment services report increases in positive parental interactions such as increases in describing and praising appropriate child behaviors (Borrego et al., 1999; Timmer et al., 2005a). Similarly, as hypothesized, there was a significantly decrease in DPICS-III Negative Talk scores from pre- to post-treatment for the PCIT group. These results also were not surprising as previous research has demonstrated that families with histories of CAN who receive PCIT treatment services report decreases in negative parental interactions such as criticizing child behaviors (Borrego et al., 1999; Timmer 2005a).

One explanation for increases in Positive Talk and decreases in Negative Talk is that these behaviors were specifically taught, modeled, and practiced in the PCIT condition. Caregivers in the PCIT condition were coached intensively in these skills. They also received frequent feedback on their progress towards skills mastery. Numerous investigations have found that, compared to didactic presentation alone, combination of coaching and feedback results in larger skill change (Kaminski, Valle, Filene, \& Boyle, 2008). While these results suggest that PCIT resulted in significant changes in parental communication styles, there was insufficient 
data to compare PCIT's effects to those of TAU. Unfortunately, the sample size for the TAU group was too small to allow for statistical comparisons of the two groups.

\section{Limitations}

Several limitations were observed during the course of this study. First, subjective assessment data were gathered via only one caregiver regarding CAN, IPV, parental stress, and child behaviors. Gathering subjective assessment data from additional sources such as caregiver's partner, child teachers, and social services may have provided more accurate information regarding a variety of child and caregiver behaviors. Objective data (DPICS-III) was gathered at pre- and post-treatment by PCIT therapists to assess some child and caregiver behaviors, but a low sample size in the TAU condition limited the usefulness of these measures. Second, the utilization of the CTS2 to assess IPV in this study was problematic. The CTS2 demonstrates face validity and participants in this study were highly motivated to portray themselves in a positive manner. As such, they may have been able to identify which questions were and were not acceptable to endorse considering their current involvement with the legal system. Furthermore, the CTS2 assessed IPV in the previous year, but did not take into account caregivers having numerous intimate partners within the year and did not assess changes in violence during the course of the relationship.

Third, skills generalization to environments outside of the therapeutic environment was not assessed. Caregivers may have utilized PCIT skills during sessions, but may not have actually implemented them across environments. Using the DPICS-III coding system to assess caregiver-child behavior at home, school, and in the community would have provided a wealth of information regarding caregivers abilities to implement PCIT skills to reinforce their children's 
appropriate behaviors and manage their children's inappropriate behaviors in more natural environments.

Fourth, not all caregivers were included in the therapeutic process. Treating merely one caregiver may result in a change in that particular caregiver-child relationship, but may not result in changes in additional caregiver-child or caregiver-caregiver relationships. For example, if only mothers participated in PCIT, an improvement in the mother-child relationship would be expected. However, an improvement in the father-child relationship would not necessarily be expected as fathers had not participated in treatment. Additionally, if only mothers are participating in treatment, then an improvement in the mother-father relationship also would not be expected as fathers had not participated in treatment. Not including the entire family in the therapeutic process may decrease the likelihood of skills generalization in CAN and IPV instances.

Fifth, child attendance was required for participation in the PCIT condition but was not a requirement for the TAU condition. Many of the children in this study were removed from the family home due to CAN and placed in fostercare or kinship care. However, child participation was required in the PCIT condition and extensive efforts were made by research assistants, DSS workers, the court system, foster parents, and kinship care placements to transport children in the PCIT condition to weekly appointments. The lack of child participation in the TAU condition was unexpected and resulted in missing data for child and caregiver behavioral observations at pre- and post-treatment. Hence, missing data coupled with issues regarding statistical power may have accounted for significantly greater statistical differences between the PCIT and TAU conditions. 
Finally, there was not a standardized protocol for the TAU treatment condition and no theoretical underpinning for topics selected as pertinent to parenting. As such, content varied dramatically between TAU groups dependent upon group membership and caregiver needs. Furthermore, TAU did not incorporate active participation, modeling of concepts, or coaching in-session for skills implementation during treatment. As a result, it is unclear whether the current TAU group is representative of standard of care for CAN in community mental health agencies across the country.

\section{Implications}

The purpose of the study was to compare two treatment groups, PCIT and TAU, in the reduction of co-morbid CAN and IPV. Preliminary findings suggest that PCIT is more effective than TAU at decreasing parental stress and negative child and caregiver behaviors, while also increasing positive child and caregiver behaviors. Unfortunately, results regarding PCIT's effectiveness at reducing recurrences of child abuse and decreasing IPV are less clear. Results from this study suggest that the group PCIT conducted in this investigation without specialized facilities or equipment may have effects similar to research studies conducting in large, university environments. These treatment effectiveness data, which suggest that PCIT can be conducted in standard community settings, are likely to have important implications for researchers and clinicians examining the transportability of evidence-based programs like PCIT into real-world settings.

\section{Future Clinical Directions}

Future clinical directions include the dissemination of research findings supporting the use of PCIT in community-based organizations as an effective treatment for family violence. This may be difficult as many community-based organizations are unfamiliar with professional 
publications and understanding research findings. Hence, it is the responsibility of research scientists to disseminate these findings in locations where community-based organizations are more likely to access the results. In addition to publishing in scientific journals, these findings could also be published in venues geared towards a more general audience such as parenting magazines. Furthermore, researchers should access local government and non-profit agencies in their areas responsible for programming to address the reduction of CAN, IPV, or both forms of family violence and provide lectures regarding research findings and how these programs may be implemented in their communities.

It is imperative that families who experience co-morbid family violence be identified quickly and enrolled in an evidence-based treatment program to help insure safety for all family members. The early implementation of evidence-based treatment programs to address and reduce co-morbid CAN and IPV and their effects may result in improved long-term outcomes for these families. This study provided evidence that families enrolled in an evidence-based program reported greater improvements in behaviors associated with family violence (e.g., parenting stress, negative communication) compared to families enrolled in a non-evidence-based program. It is crucial that organizations provide the best services possible for families in their communities.

Conducting PCIT in a group format may have additional benefits compared to conducting PCIT in an individual format. Caregivers and children in this study not only demonstrated results similar to studies conducted individually, but also appeared to benefit from having other caregivers as part of their social support structure. In addition, conducting PCIT in groups was extremely cost- and time-efficient which is an important consideration for community-based organizations with limited resources. 
Organizations who elect to provide PCIT treatment services to populations affected by CAN and/or IPV may wish to incorporate several program modifications to improve treatment results: (a) including 1-2 sessions during the course of treatment that specifically focus on addressing the short- and long-term effects of CAN and IPV upon families, (b) including program content directly addressing violence in the caregiver-caregiver relationship, (c) including program content directed towards teaching the children about CAN and developing a safety plan for future incidences, (d) giving the children into more active roles in the PCIT treatment process such as teaching them PCIT skills and requiring them to implement these skills with their caregivers or siblings, and (e) considering the role of culture in the implementation of PCIT treatment components and how PCIT protocols could be tailored to accommodate cultural differences across families. While all of these clinical modifications have a strong theoretical base, future research still is required to determine the ultimate impact that these changes may have on remediating family violence.

\section{Future Research Directions}

Future research directions include the designing and implementation of large-scale, longitudinal research studies that explore the impact of PCIT on co-morbid CAN and IPV populations. Of particular interest would be follow-up studies that assess the retention of skills taught during PCIT treatment. Additionally, it is not only important to use measures of child abuse potential but to follow up with families regarding actual rates of CAN and IPV recidivism. Similarly, future researchers should consider gathering data from other objective sources, such as court- or medical-records, to gauge the frequency and severity of CAN and IPV.

Assessing PCIT skills generalization outside of the therapeutic environment is crucial to evaluating the effectiveness of PCIT across natural environments. While it is neither time- or 
cost-efficient to conduct DPICS-III coding at home and in the community, this data would provide extensive information regarding caregivers' abilities to implement the skills learned during session to other environments. One affordable method of assessing skills generalization may be to have families audiotape their daily PCIT homework. This assessment method would allow researchers to assess for homework compliance, the number of days PCIT is practiced, the total number of minutes caregivers practice these skills per week, the mastery of PCIT skills in the home environment, and aspects of the household environment and specific barriers towards implementing these skills in natural environments. Furthermore, in order to assess IPV more accurately and whether PCIT skills generalized to the caregiver-caregiver relationship, it may be beneficial to observe a caregiver-caregiver interaction where the couple is provided with a predetermined activity, such as a complicated puzzle. A coding system could be used to quantify their verbal interactions at pre- and post-treatment in order to assess if they are using PCIT skills in their relationship.

In conclusion, the current study has many implications for community treatment of family violence. The research provides promising results regarding the use of PCIT in a group format when working with caregivers who physically abuse their children. The study also provided important information suggesting that results can be obtained in typical community agencies without the addition of one-way mirrors, communication devices, and specially constructed rooms. Finally, this research illuminates many of the methodological issues that need to be addressed to further our knowledge with regard to the potential importance of PCIT in cases of family violence. 


\section{References}

Abidin, R.R. (1995). Parenting Stress Index, $3^{\text {rd }}$ Ed. Psychological Assessment Resources, Inc. Lutz, FL.

Achenbach, T. (1990). Young adult behavior checklist. Burlington, VT: University of Vermont Department of Psychiatry.

Achenbach, T.M. (2001). Manual for the ASEBA School-Age Forms and Profiles. Burlington, VT. University of Vermont, Research Center for Children, Youth \& Families.

Achenbach, T.M., \& Rescorla, L. (2000). Manual for the ASEBA Preschool Forms \& Profiles. Burlington, VT. University of Vermont, Research Center for Children, Youth \& Families. American Psychiatric Association. (2005). What is domestic violence? Informational Materials, $1-4$.

Appel, A.E., \& Holden, G.W. (1998). The co-occurrence of spouse and physical child abuse: A review and appraisal. Journal of Family Psychology, 12, 4, 578-599.

Arias, I. (2004). The legacy of child maltreatment: Long-term health consequences for women. Journal of Women's Health, 13, 5, 468-473.

Babcock, J.C., Green, C.E., \& Robie, C. (2004). Does batterer treatment work? A meta-analytic review of domestic violence treatment. Clinical Psychology Review, 23, 1023-1053.

Bagner, D.M., \& Eyberg, S.M. (2003). Father involvement in parent training: When does it matter? Journal of Clinical Child Psychology, 32, 4, 599-605.

Bagner, D.M., \& Eyberg, S.M. (2007). Parent-Child Interaction Therapy for disruptive behavior in children with mental retardation: A randomized controlled trial. Journal of Clinical Child and Adolescent Psychology, 36, 418-429.

Baumrind, D. (1967). Child care practices anteceding three patterns of preschool behavior. 
Generic Psychology Monographs, 75, 43-88.

Bell, S., \& Eyberg, S.M. (2002). Parent-Child Interaction Therapy. In L. VandeCreek, S. Knapp, \& T.L. Jackson (Eds.). Innovations in Clinical Practice: A Source Book (Vol. 20; pp. 5774). Sarasota, FL: Professional Resource Press.

Boden, J.M., Horwood, L.J., \& Fergusson, D.M. (2007). Exposure to childhood sexual and physical abuse and subsequent educational achievement outcomes. Child Abuse \& Neglect, 31, 1101-1114.

Boggs, S.R., Eyberg, S.M., Edwards, D.L., Rayfield, A., Jacobs, J., Bagner, D., \& Hood, K.,K. (2004). Outcomes of Parent-Child Interaction Therapy: A comparison of treatment completers and study dropouts one to three years later. Child \& Family Behavior Therapy, 26, 4, 1-22.

Borrego, J.J., Gutow, M.R., Reicher, S., \& Barker, C.H. (2008). Parent-Child Interaction Therapy with domestic violence populations. Journal of Family Violence, 23, 495-505.

Borrego, J.J., Urquiza, A.J., Rasmussen, R.A., \& Zebell, N. (1999). Parent-Child Interaction Therapy with a family at high risk for physical abuse. Child Maltreatment, 4, 4, 331-342.

Bowlby, J., \& Ainsworth, M.D.S. (1991), An ethological approach to personality development. American Psychologist, 46, 331-341.

Brestan, E.V., Eyberg, S.M., Boggs, S.R., \& Algina, J. (1997). Parent-child interaction therapy: Parent perceptions of untreated siblings. Child \& Family Behavior Therapy, 19, 13-28.

Brestan, E.V., \& Eyberg, S.M. (1998). Effective psychosocial treatments for children and adolescents with disruptive behavior disorders: 29 years, 82 studies, and 5272 kids. Journal of Clinical Child Psychology, 27, 179-188.

Brestan, E., Jacobs, J., Rayfield, A., \& Eyberg, S.M. (1999). A consumer satisfaction measure 
for parent-child treatments and its relationship to measures of child behavior change. Behavior Therapy, 30, 17-30.

Brinkmeyer, M., \& Eyberg, S.M. (2003). Parent-child interaction therapy for oppositional children. In A.E. Kazdin \& J.R. Weisz (Eds.). Evidence-based psychotherapies for children and adolescents (pp. 204-223). New York: Guilford.

Calzada, E., Eyberg, S.M., Rich, B., \& Querido, J.G. (2004). Parenting disruptive preschoolers: Experience of mothers and fathers. Journal of Abnormal Child Psychology, 32, 203-213. Chadwick Center (2004). Closing the quality chasm in child abuse treatment: Identifying and disseminating best practices: Findings of the Kauffman best practices project to help children heal from child abuse. San Diego, CA: Children's Hospital-San Diego, Kauffman Center for Children and Families.

Chaffin, M., Silovsky, J.F., Funderbunk, B., Valle, L.A., Brestan, E.V., Alachua, T., Jackson, S., Lensgraf, J, \& Bonner, B.L. (2004). Parent-Child Interaction Therapy with physically abusive parents: Efficacy for reducing future abuse reposts. Journal of Consulting and Clinical Psychology, 72, 3, 500-510.

Choate, M.L., Pincus, D.B., Eyberg, S.E., \& Barlow, D.H. (2005). Parent-Child Interaction Therapy for treatment of Separation Anxiety Disorder in young children: A pilot study. Cognitive and Behavioral Practice, 12, 126-135.

Cohen, J. (1992). A power primer. Psychological Bulletin, 112, 1, 155-159.

Cohen, J. (1988). Statistical power analysis for the behavioral sciences ( $2^{\text {nd }}$ edition). Hillsdale, NJ: Erlbaum.

Cohen, J.A., Mandarin, A.P., Murray, L.K., \& Gellman, R. (2006). Psychosocial Interventions for maltreated and violence-exposed children. Journal of Social Issues, 62, 4, 737-766. 
DePanfilis, D., \& Zuravin, S.J. (2002). The effect of services on recurrence of child maltreatment. Child Abuse and Neglect, 26, 187-206.

Derogatis, L.R. (1993). The Brief Symptom Inventory (BSI): Administration, scoring and procedures manual. Minneapolis, MN: Pearson Inc.

Derogatis, L.R. \& Melisaratos, N. (1983). The Brief Symptom Inventory: An introductory report. Psychological Medicine, 13, 3, 595-605.

Dombrowski, S.C., Timmer, S.G., Blacker, D.M., \& Urquiza, A.J. (2005). A positive behavioural intervention for toddlers: Parent-Child Attunement Therapy. Child Abuse Review, 14, 132-151.

Edleson, J.L. \& Syers, M. (1990). Relative effectiveness of group treatments for men who batter. Social Work Research \& Abstracts, 26, 2, 10-17.

Eyberg, S.M. (1988). Parent-child interaction therapy: Integration of traditional and behavioral concerns. Child \& Family Behavior Therapy, 10, 33-46.

Eyberg, S.M. (1993). Consumer satisfaction measures for assessing parent training programs. In L. VandeCreek, S. Knapp, \& T.L. Jackson (Eds.). Innovations in clinical practice: A source book (Vol. 12). Sarasota, FL: Professional Resource Press.

Eyberg, S.M. (2004). The PCIT story part 1: Conceptual foundation. PCIT Pages: The ParentChild Interaction Therapy Newsletter, 1, 1-2.

Eyberg, S.M. (2005). Tailoring and adapting Parent-Child Interaction Therapy to new populations. Education and Treatment of Children, 28, 2, 197-201.

Eyberg, S.M., \& Johnson, S.M. (1974). Multiple assessment of behavior modification with families: Effects of contingency contracting and order of treated problems. Journal of Consulting and Clinical Psychology, 42, 594-606. 
Eyberg, S.M., McDiarmid Nelson, M., Duke, M., \& Boggs, S.R. (2004). Manual for the Dyadic Parent-Child Interaction Therapy Coding System, Third Edition. Unpublished manuscript. University of Florida.

Eyberg, S. \& Members of the Child Study Laboratory. (1999). Parent-Child Interaction Therapy Treatment Manual. Integrity Checklists and Session Materials. Unpublished manuscript. University of Florida.

Eyberg, S., \& Pincus, D. (1999). Eyberg Child Behavior Inventory \& Sutter-Eyberg Student Behavior Inventory - Revised. Psychological Assessment Resources, Odessa, FL: Psychological Assessment Resources.

Eyberg, S.M., \& Robinson, E.A. (1982). Parent-child interaction training: Effects on family functioning. Journal of Clinical Child Psychology, 11, 2, 130-137.

Faul, F., Erdfelder, E., Lang, A.G., \& Buchner, A. (2007). G*Power 3: A flexible statistical power analysis program for the social, behavioral, and biomedical sciences. Behavior Research Methods, 39, 175-191.

Greco, L.A., Sorrell, J.T., \& McNeil, C.B. (2001). Understanding manual-based behavior therapy: Some theoretical foundations of parent-child interaction therapy. Child \& Family Behavior Therapy, 23, 21-36.

Hanf, C. A. (1969). A two-stage program for modifying maternal controlling during motherchild $(M-C)$ interaction. Paper presented at the meeting of the Western Psychological Association, Vancouver.

Harris, G.E. (2006). Conjoint therapy and domestic violence: Treating the individuals and the relationship. Counseling Psychology Quarterly, 19, 4, 373-379.

Harwood, M., \& Eyberg, S.M. (2004). Therapist Verbal Behavior Early in Treatment: Relation 
to Successful Completion of Parent-Child Interaction Therapy. Journal of Clinical Child and Adolescent Psychology, 33, 601-612.

Hembree-Eisenstadt, T., Eyberg, S., McNeil, C.B., Newcomb, K., \& Funderburk. B. (1993). Parent-child Interaction Therapy with behavior problem children: Relative effectiveness of two stages and overall treatment outcome. Journal of Clinical Child Psychology, 22, 1, 42-51.

Herrenkohl, T.I., \& Herrenkohl, R.C. (2007). Examining the overlap and prediction of multiple forms of child maltreatment, stressors, and socioeconomic status: A longitudinal analysis of youth outcome. Journal of Family Violence, 22, 553-562.

Herschell, A.D., Calzada, E.J., Eyberg, S.M., \& McNeil, C.B. (2002). Clinical issues in Parent-Child Interaction Therapy. Cognitive and Behavioral Practice, 9, 16, 16-27.

Herschell, A.D., \& McNeil, C.B. (2005). Theoretical and empirical underpinnings of ParentChild Interaction Therapy with child physical abuse populations. Education and Treatment of Children, 28, 2, 142-162.

Hembree-Kigin, T., \& McNeil, C. (1995). Parent-Child Interaction Therapy. New York: Plenum. Hollingshead, A. (1975). Four factor index of social status. Unpublished manuscript. New Haven, CT: Yale University.

Lang, A.J., Stein, M.B., Kennedy, C.M., \& Foy, D.W. (2004). Adult psychopathology and intimate partner violence among survivors of childhood maltreatment. Journal of Interpersonal Violence, 19, 10, 1102-1118.

Lieberman, A.F., \& Knorr, K. (2007). The impact of trauma: A developmental framework for infancy and early childhood. Psychiatric Annals, 37, 6, 416-422.

Makhija, N.J. (2007). Childhood abuse and adolescent suicidality: A direct link and an indirect 
link through alcohol and substance misuse. International Journal of Adolescent Medicine and Health, 19, 1, 45-51.

Matos, M., Torres, R., Santiago, R., Jurado, M., \& Rodriguez, I. (2006). Adaptation of ParentChild Interaction Therapy for Puerto Rican families: A preliminary study. Family Process, 45, 2, 205-222.

McDiarmid, M.D., \& Bagner, D.M. (2005). Parent-Child Interaction Therapy for children with disruptive behavior and developmental disabilities. Education and Treatment of Children, $28,130-141$.

McNeil, C.B., Filcheck, H.A., Greco, L.A., Ware, L.M., \& Bernard, R.S. (2001). Parent-Child Interaction Therapy: Can a manualized treatment be functional? The Behavior Analyst Today, 2, 106-114.

Milner, J.S. (1986). CAP Inventory Manual, $2^{\text {nd }}$ Ed., An Interpretive Manual for the CAP Inventory. Psychological Assessment Resources, Lutz, FL.

Niec, L.N., Hemme, J.M., Yopp, J., \& Brestan, E.V. (2005). Parent-Child Interaction Therapy: The rewards and challenges of a group format. Cognitive and Behavioral Practice.

Nixon, Sweeney, \& Erickson. (2004). Parent-Child Interaction Therapy: Oneand two-year follow-up of standard and abbreviated treatments for oppositional Preschoolers. Journal of Abnormal Child Psychology, 32, 3, 263-271.

O’Leary, D.K. (2002). Conjoint therapy for partners who engage in physically aggressive behavior: Rationale and research. Journal of Aggression, Maltreatment \& Trauma, 5, 2, $145-164$.

Patterson, G.R. (1982). Coercive family process. Eugene, OR: Castalia. Pearl, E.S. (2008). Parent-Child Interaction therapy with an immigrant family exposed to 
domestic violence. Clinical Case Studies, 7, 1, 25-41.

Pincus, D.B., Eyberg, S.M., \& Choate, M.L. (2005). Adapting Parent-Child Interaction Therapy for young children with Separation Anxiety Disorder. Education and Treatment of Children, 28, 2, 163-181.

Schuhmann, E.M., Foote, R.C., Eyberg, S.M., Boggs, S.R., \& Algina, J. (1998). Efficacy of Parent-Child Interaction Therapy: Interim report of a randomized trial with short-term maintenance. Journal of Clinical Child Psychology, 27, 1, 34-45.

Springer, K.W., Sheridan, J., Kuo, D., \& Carnes, M. (2007). Long-term physical and mental health consequences of childhood physical abuse: Results from a large population-based sample of men and women. Child Abuse \& Neglect, 31, 517-530.

Sternberg, K.J., Lamb, M.E., Guterman, E., \& Abbott, C.B. (2006). Effects of early and later family violence on children's behavior problems and depression: A longitudinal, multiinformant perspective. Child Abuse \& Neglect, 30, 283-306.

Straus, M.A., Hamby, S.L., Boney-McCoy, S., \& Sugarman, D.B. (1996). The Revised Conflict Tactics Scale (CTS2-form A). Family Research Laboratory, Durham, NH.

Tjaden, P., \& Tiioennes, N. (2002). Full Report of the Prevalence, Incidence, and Consequences of Violence Against Women: Findings from the National Violence Against Women Survey, Washington, DC: Department of Justice.

Thomas, R., \& Zimmer-Gembeck, M.J. (2007). Behavioral outcomes of Parent-Child Interaction Therapy and Triple P - Positive Parenting Program: A review and meta-analysis, Journal of Abnormal Child Psychology, 35, 475-495.

Timmer, S.G., Borrego, J., \& Urquiza, A.J. (2002). Antecedents of coercive interactions in physically abusive mother-child dyads. Journal of Interpersonal Violence, 17, 8, 836- 
853.

Timmer, S.G., Urquiza, A.J., \& Zebell, N. (2005a). Challenging foster caregiver-maltreated child relationships: The effectiveness of parent-child interaction therapy. Children and Youth Services Review, 28, 1-19.

Timmer, S.G., Urquiza, A.J., Zebell, N.M., \& McGrath, J.M. (2005b). Parent-Child Interaction Therapy: Application to maltreating parent-child dyads. Child Abuse \& Neglect, 29, 825-842.

Timmer, S.G., Urquiza, A.J., Herschell, A.D., McGrath, J.M., Zebell, N.M., Porter, A.L., \& Vargas, E.C. (2006). Parent-Child Interaction Therapy: Application of an empirically supported treatment to maltreated children in foster care. Child Welfare League of America, 919-939.

Urquiza, A.J., \& McNeil, C.B. (1996). Parent-Child Interaction Therapy: An intensive dyadic intervention for physically abusive families. Child Maltreatment, 1, 2, 134-144.

U.S. Department of Health \& Human Services, Administration on Children, Youth, and Families. Child Maltreatment 2005. (Washington, DC: U.S. Government Printing Office, 2007).

U.S. Department of Health \& Human Services, Administration on Children, Youth, and Families. The Child Abuse Prevention and Treatment Act. (Washington, DC: U.S. Government Printing Office, 2003).

Ware, L.M., Fortson, B.L., \& McNeil, C.B. (2003). Parent-Child Interaction Therapy: A promising Intervention for abusive families. The Behavior Analyst Today, 3, 375-382. Werba, B.E., Eyberg, S.M., Boggs, S.R. \& Algina, M. (2006). Predicting outcome in Parent- 
Child Interaction Therapy: Successes and attrition. Behavior Modification, 30, 5, 618646.

Wierzbicki, M. \& Pekarik, G. (1993). A meta-analysis of psychotherapy dropout. Professional Psychology: Research and Practice, 24, 190-195. 
Table 1.

Demographic information

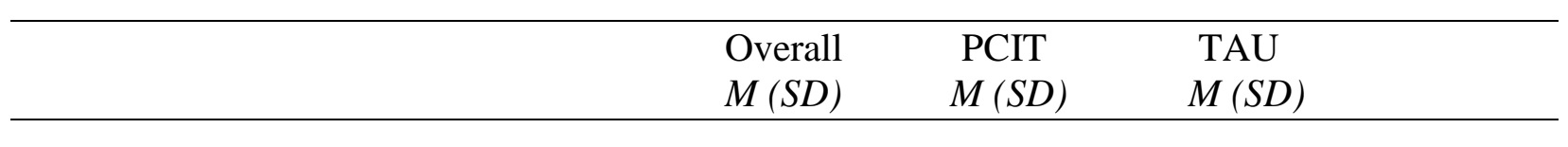

Number of Participants

Pre-treatment

Post-treatment
49

43

Number of Sessions Attended

0 Sessions

1 Session

2 Sessions

3 Sessions

4 Sessions

5 Sessions

6 Sessions

7 Sessions

8 Sessions

9 Sessions

10 Sessions

11 Sessions

12 Sessions

Missing Data

Average Number of Sessions (SD)

$\begin{array}{lll}9 & 21 & 28 \\ 3 & 19 & 24\end{array}$

$\begin{array}{lll}1 & 0 & 1 \\ 4 & 1 & 3 \\ 1 & 1 & 0 \\ 2 & 1 & 1 \\ 5 & 1 & 4 \\ 0 & 0 & 0 \\ 4 & 0 & 4 \\ 3 & 1 & 2 \\ 6 & 3 & 3 \\ 14 & 5 & 9 \\ 5 & 5 & 0 \\ 2 & 2 & 0 \\ 4 & 4 & 0 \\ 2 & 1 & 1 \\ 6.96(3.19) & 8.10(3.18) & 6.11(3.00)\end{array}$

Adult Participants

Gender

Female

Male

Ethnicity

Caucasian

African-American

No Reported

Age

Female

Male
49

41

08

49

46

02

01

$30.29(7.45) \quad 28.32(6.85)$

$35.36(10.68) \quad 34.44(9.39) \quad 35.92(12.43)$ 
Relationship

Cohabitating

Divorce

Married

Re-Married

Separated

Single

SES

Category 1

Category 2

Category 3

Category 4

Category 5

Not Reported

Education

Junior High School

Partial High School

High School or GED

Partial College

College Graduate

Professional Degree

Brief Symptom Inventory

Global Severity Index
Child Participants

Gender

Female

Male

Ethnicity

Caucasian

Multiple Ethnicities

Age

Female

Male
49

14

06

14

02

07

06

$\begin{array}{lll}1.70(0.95) & 1.50(0.51) & 1.85(1.17) \\ 25 & 10 & 14 \\ 18 & 10 & 08 \\ 01 & 00 & 01 \\ 03 & 00 & 03 \\ 01 & 00 & 01 \\ 02 & 01 & 01\end{array}$

49

03

13

17

13

00

01

$60.92(11.37) 60.29(11.49) 61.39(11.47)$
28

08

03

06

02

05

$02 \quad 04$

$21 \quad 28$

$00 \quad 03$

$07 \quad 06$

$07 \quad 10$

$06 \quad 07$

$01 \quad 01$

$00 \quad 01$

28
03
0
0
07
01


Table 2.

Overall scores of pre-and post-treatment assessment measures

\begin{tabular}{|c|c|c|}
\hline & $\begin{array}{c}\text { Pre-treatment } \\
\qquad M(S D)\end{array}$ & $\begin{array}{c}\text { Post-treatment } \\
M(S D)\end{array}$ \\
\hline CAP Physical Child Abuse & $171.36(113.84)$ & $154.48(107.23)$ \\
\hline CTS2 Female Psychological Violence & $16.63(17.34)$ & $18.85(22.49)$ \\
\hline CTS2 Male Psychological Violence & $20.00(23.83)$ & $19.85(25.22)$ \\
\hline CTS2 Female Physical Violence & $2.65(5.98)$ & $6.29(29.29)$ \\
\hline CTS2 Male Physical Violence & $5.60(25.10)$ & $3.39(7.27)$ \\
\hline PSI-SF Total Stress & $88.02(29.37)$ & $77.02(21.90)$ \\
\hline CBCL Internalizing & $57.00(11.26)$ & $52.10(12.39)$ \\
\hline CBCL Externalizing & $59.86(11.15)$ & $55.02(11.58)$ \\
\hline CBCL Total & $58.40(13.09)$ & $52.79(13.88)$ \\
\hline Child Compliance & ----------------- & $0.87(0.26)$ \\
\hline ECBI Intensity & $118.71(45.27)$ & $90.98(43.76)$ \\
\hline ECBI Problem & $13.68(11.62)$ & $9.59(11.13)$ \\
\hline DPICS-III Positive Talk & $11.89(6.69)$ & $18.89(12.46)$ \\
\hline DPICS-III Negative Talk & $7.02(4.75)$ & $5.32(7.97)$ \\
\hline
\end{tabular}


Parent Child Interaction Therapy 88

Table 3.

Repeated-measures ANCOVA of CAP physical child abuse scores over treatment, based upon treatment condition

\begin{tabular}{lcccc}
\hline & $\begin{array}{c}\text { Pre-treatment } \\
M(S D)\end{array}$ & $\begin{array}{c}\text { Post-treatment } \\
M(S D)\end{array}$ & $d$ & Effect Size \\
\hline PCIT & $144.95(94.75)$ & $111.60(76.93)^{* *}$ & 0.39 & 0.19 \\
TAU & $190.93(124.24)$ & $190.21(116.95)^{* *}$ & 0.01 & 0.00 \\
\hline$* * p>.05$ & & & &
\end{tabular}


Parent Child Interaction Therapy 89

Table 4.

Repeated-measures ANCOVA of CTS2 male-to-female psychological violence scores over treatment, based upon treatment condition

\begin{tabular}{lcccc}
\hline & $\begin{array}{c}\text { Pre-treatment } \\
M(S D)\end{array}$ & $\begin{array}{c}\text { Post-treatment } \\
M(S D)\end{array}$ & $d$ & Effect Size \\
\hline PCIT & $17.50(14.88)$ & $14.21(17.66)$ & 0.20 & 0.10 \\
TAU & $15.87(19.53)$ & $22.86(25.68)$ & -0.31 & -0.15 \\
\hline$p>.05$ & & & &
\end{tabular}


Parent Child Interaction Therapy 90

Table 5.

Repeated-measures ANCOVA of CTS2 female-to-male psychological violence scores over treatment, based upon treatment condition

\begin{tabular}{lcccc}
\hline & $\begin{array}{c}\text { Pre-treatment } \\
M(S D)\end{array}$ & $\begin{array}{c}\text { Post-treatment } \\
M(S D)\end{array}$ & $d$ & Effect Size \\
\hline PCIT & $19.55(18.42)$ & $13.53(19.70)$ & 0.32 & 0.16 \\
TAU & $20.39(28.12)$ & $25.32(28.47)$ & -0.17 & -0.09 \\
\hline$p>.05$ & & & &
\end{tabular}


Parent Child Interaction Therapy 91

Table 6.

Repeated-measures ANCOVA of CTS2 male-to-female physical violence scores over treatment, based upon treatment condition

\begin{tabular}{lcccc}
\hline & $\begin{array}{c}\text { Pre-treatment } \\
M(S D)\end{array}$ & $\begin{array}{c}\text { Post-treatment } \\
M(S D)\end{array}$ & $d$ & Effect Size \\
\hline PCIT & $3.55(7.23)$ & $1.53(3.32)$ & 0.36 & 0.18 \\
TAU & $1.87(4.66)$ & $0.41(39.83)$ & -0.30 & -0.15 \\
\hline$p>.05$ & & & &
\end{tabular}


Parent Child Interaction Therapy 92

Table 7.

Repeated-measures ANCOVA of CTS2 female-to-male physical violence scores over treatment, based upon treatment condition

\begin{tabular}{lcccc}
\hline & $\begin{array}{c}\text { Pre-treatment } \\
M(S D)\end{array}$ & $\begin{array}{c}\text { Post-treatment } \\
M(S D)\end{array}$ & $d$ & Effect Size \\
\hline PCIT & $9.85(36.44)$ & $2.05(5.68)$ & 0.30 & 0.15 \\
TAU & $1.91(4.97)$ & $4.55(8.35)$ & -0.38 & -0.19 \\
\hline$p>.05$ & & & &
\end{tabular}


Parent Child Interaction Therapy 93

Table 8.

Repeated-measures ANCOVA of PSI-SF total stress score over treatment, based upon treatment condition

\begin{tabular}{lcccc}
\hline & $\begin{array}{c}\text { Pre-treatment } \\
M(S D)\end{array}$ & $\begin{array}{c}\text { Post-treatment } \\
M(S D)\end{array}$ & $d$ & Effect Size \\
\hline PCIT & $95.35(33.10)$ & $72.25(17.73)^{* *}$ & 0.87 & 0.40 \\
TAU & $82.59(25.57)$ & $81.00(24.51)^{* *}$ & 0.06 & 0.03 \\
$* * p<.05$ & & & &
\end{tabular}


Parent Child Interaction Therapy 94

Table 9.

Repeated-measures ANCOVA of CBCL internalizing behavior problem scores over treatment, based upon treatment condition

\begin{tabular}{lcccc}
\hline & $\begin{array}{c}\text { Pre-treatment } \\
M(S D)\end{array}$ & $\begin{array}{c}\text { Post-treatment } \\
M(S D)\end{array}$ & $d$ & Effect Size \\
\hline PCIT & $80.00(61.47)^{* * *}$ & $73.00(52.11)^{* * *}$ & 0.83 & 0.38 \\
TAU & $74.00(53.46)^{* * *}$ & $74.00(52.09)^{* * *}$ & 0.12 & 0.08 \\
$* * * p<.05, * * * p<.001$ & & &
\end{tabular}


Parent Child Interaction Therapy 95

Table 10.

Repeated-measures ANCOVA of CBCL externalizing behavior problem scores over treatment, based upon treatment condition

\begin{tabular}{lcccc}
\hline & $\begin{array}{c}\text { Pre-treatment } \\
M(S D)\end{array}$ & $\begin{array}{c}\text { Post-treatment } \\
M(S D)\end{array}$ & $d$ & Effect Sizes \\
\hline PCIT & $63.05(10.85)$ & $55.37(12.85)^{* *}$ & 0.65 & 0.31 \\
TAU & $57.33(10.95)$ & $54.74(10.71)^{* *}$ & 0.24 & 0.11 \\
$* * p>.05$ & & & &
\end{tabular}


Parent Child Interaction Therapy 96

Table 11.

Repeated-measures ANCOVA of CBCL total behavior problem scores over treatment, based upon treatment condition

\begin{tabular}{lcccc}
\hline & $\begin{array}{c}\text { Pre-treatment } \\
M(S D)\end{array}$ & $\begin{array}{c}\text { Post-treatment } \\
M(S D)\end{array}$ & $d$ & Effect Size \\
\hline PCIT & $61.74(11.53)$ & $52.41(14.48)^{* *}$ & 0.71 & 0.34 \\
TAU & $55.75(13.82)$ & $53.09(13.68)^{* *}$ & 0.19 & 0.10 \\
$* * p>.05$ & & & &
\end{tabular}


Table 12.

Description of Child Compliance scores, based upon treatment condition

\begin{tabular}{lcc}
\hline & $\begin{array}{c}\text { Pre-treatment } \\
M(S D)\end{array}$ & $\begin{array}{c}\text { Post-treatment } \\
M(S D)\end{array}$ \\
\hline Parent-Child Interaction Therapy & -------------- & $0.90(0.25)$ \\
Treatment as Usual & $1.00(0.00)$ & $0.68(0.28)$ \\
\hline
\end{tabular}


Parent Child Interaction Therapy 98

Table 13.

Repeated-measures ANCOVA of ECBI Intensity scores over treatment, based upon treatment condition

\begin{tabular}{lcccc}
\hline & $\begin{array}{c}\text { Pre-treatment } \\
M(S D)\end{array}$ & $\begin{array}{c}\text { Post-treatment } \\
M(S D)\end{array}$ & $d$ & Effect Size \\
\hline PCIT & $133.26(49.92)$ & $89.65(46.04) * * *$ & 0.91 & 0.41 \\
TAU & $108.08(39.17)$ & $92.08(42.73) * * *$ & 0.39 & 0.19 \\
& & & &
\end{tabular}


Parent Child Interaction Therapy 99

Table 14.

Repeated-measures ANCOVA of ECBI Problem scores over treatment, based upon treatment condition

\begin{tabular}{lcccc}
\hline & $\begin{array}{c}\text { Pre-treatment } \\
M(S D)\end{array}$ & $\begin{array}{c}\text { Post-treatment } \\
M(S D)\end{array}$ & $d$ & Effect Size \\
\hline PCIT & $16.63(13.11)$ & $8.25(9.35)^{* *}$ & 0.74 & 0.35 \\
TAU & $11.44(10.04)$ & $10.71(12.50)^{* *}$ & 0.06 & 0.03 \\
$* * p<.01$ & & & &
\end{tabular}


Table 15.

Paired samples t-test of DPICS-III Positive Talk scores over treatment for PCIT

\begin{tabular}{lcc}
\hline & $\begin{array}{c}\text { Pre-treatment } \\
M(S D)\end{array}$ & $\begin{array}{c}\text { Post-treatment } \\
M(S D)\end{array}$ \\
\hline Parent-Child Interaction Therapy & $12.88(6.62)$ & $21.75(11.43)^{* *}$ \\
\hline$* * p<.01$ & &
\end{tabular}


Table 16.

Independent samples t-test of DPICS-III Negative Talk scores over treatment for PCIT

\begin{tabular}{lcc}
\hline & $\begin{array}{c}\text { Pre-treatment } \\
M(S D)\end{array}$ & $\begin{array}{c}\text { Post-treatment } \\
M(S D)\end{array}$ \\
\hline Parent-Child Interaction Therapy & $6.03(4.19)$ & $2.44(2.03) * * *$ \\
\hline$* * p>.000$ & &
\end{tabular}


Table 17.

Caregiver report of CTS2 over treatment

\begin{tabular}{lcccc}
\hline & \multicolumn{2}{c}{ Pre-treatment } & \multicolumn{2}{c}{ Post-treatment } \\
\hline & $\begin{array}{c}\text { Not } \\
\text { Present }\end{array}$ & Present & $\begin{array}{c}\text { Not } \\
\text { Present }\end{array}$ & Present \\
\hline Male-to-female physical & $65.1 \%$ & $34.9 \%$ & $65.9 \%$ & $34.1 \%$ \\
Female-to-male physical & $74.4 \%$ & $25.6 \%$ & $68.3 \%$ & $31.7 \%$ \\
Male-to-female negotiation & $9.3 \%$ & $90.7 \%$ & $7.3 \%$ & $92.7 \%$ \\
Female-to-male negotiation & $4.7 \%$ & $95.3 \%$ & $7.3 \%$ & $92.7 \%$ \\
Male-to-female injury & $86.0 \%$ & $14.0 \%$ & $90.2 \%$ & $9.8 \%$ \\
Female-to-male injury & $93.0 \%$ & $7.0 \%$ & $87.8 \%$ & $12.8 \%$ \\
Male-to-female psychological & $14.0 \%$ & $86.0 \%$ & $22.0 \%$ & $78.0 \%$ \\
Female-to-male psychological & $20.9 \%$ & $79.1 \%$ & $24.4 \%$ & $74.6 \%$ \\
Male-to-female sexual & $88.4 \%$ & $11.6 \%$ & $87.8 \%$ & $12.2 \%$ \\
Female-to-male sexual & $81.4 \%$ & $18.6 \%$ & $82.9 \%$ & $17.1 \%$ \\
\hline
\end{tabular}

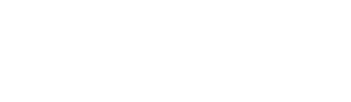

